\title{
The impulse approximation for electron transfer in reactive nucleus-atom collisions
}

\author{
D H Jakubassa-Amundsen \\ Physics Section, University of Munich, W-8046 Garching, Federal Republic of Germany
}

Received 7 December 1990, in final form 12 March 1991

\begin{abstract}
The influence of nuclear reactions on the capture of a target electron by a light, energetic particle is described within a quantum mechanical model which combines the impulse approximation for electron capture with a two-channel formalism for the nuclear reaction. Capture probabilities are calculated for $\left(p, p^{\prime}\right),(p, \alpha),(d, p)$ and $(n, \alpha)$ reactions and compared with the results for elastic nuclear scattering. With the exception of the $(n, \alpha)$ reaction, large excursions of the capture probabilities are found when the collision energy is varied across the nuclear resonance. In many cases, the excursions in the reaction channel exceed those from the elastic channel.
\end{abstract}

\section{Introduction}

The phenomenon of nuclear interference effects on atomic transition probabilities has attracted considerable interest (Heinz 1987). These interference effects are caused by the transient formation of a compound nucleus during the collision, which leads to a time delay between the electronic excitations on the ingoing and outgoing parts of the collision. A necessary condition for the occurrence of structures in the transition probability is the matching of the nuclear decay width with the energy transferred to the active electron. The applicability of the interference phenomenon extends from the measurement of nuclear lifetimes to an experimental test of atomic theories, as concerns, e.g. half-trajectory transition amplitudes or phase differences.

The first theoretical approaches for evaluating the effect of resonances in the elastic nuclear channel on the probability for target ionization date back to Blair and Anholt (1982), Feagin and Kocbach (1981) and McVoy and Weidenmüller (1982). Within the first-order Born approximation for ionization, the theory has recently been refined by Amundsen and Aashamar (1986). For electron capture, the influence of an elastic resonance has been studied in the framework of the strong potential Born approximation (Amundsen and Jakubassa-Amundsen 1984a, Jakubassa-Amundsen and Amundsen 1985). In all these approaches, the presence of a nuclear resonance manifests itself basically in the appearance of the strongly energy-dependent nuclear scattering amplitude in any first- or higher-order contribution to the atomic transition amplitude. The factorization into the scattering amplitude and the electronic amplitude for ionization or capture is made possible by the different length scales of atomic and nuclear processes, such that in the region where the atomic transitions predominantly occur, the nuclear wavefunctions have acquired their asymptotic form. 
An extension of these processes to allow for nuclear reactions, such as excitation of the target nucleus or nucleon transfer reactions, provides considerably more insight into the collision phenomena. Due to the energy defect of the reaction, the collision velocities in the incoming and outgoing parts of the collision will in most cases deviate appreciably from each other, and an interpretation of the interference structures in terms of a simple phase difference between the corresponding atomic amplitudes (Dost et al 1985) will no longer be possible. Moreover, when proton transfer is involved, the perturbative atomic potentials before and after the nuclear reaction will be different, and there arises the question whether one of the atomic amplitudes may be suppressed, such that the resulting transition probability actually is a 'half-trajectory' transition probability (Horsdal Pedersen 1987).

Theoretical attempts to generalize the ionization theory to include nuclear reactions have been set forth by Tomoda (1984) for the special case of nuclear excitation and by Anholt (1985) for arbitrary reactions. A more rigorous formulation for arbitrary reactions has been introduced by Amundsen (1986), which is based on a two-channel approach for the nuclear reaction (Amundsen and Jakubassa-Amundsen 1991).

This work provides a generalization of the charge transfer theory, making use of the nuclear two-channel approach which has been adapted-to allow for higher-order atomic theories. For the description of the atomic process, the impulse approximation (IA; McDowell and Coleman 1970) is applied, which is the on-shell limit of the strong potential Born (SPB) theory. Compared with the SPB, the IA is more readily accessible to peaking approximations which are less restrictive than the one used in the onechannel SPB calculation (Jakubassa-Amundsen and Amundsen 1985), because of the absence of additional strongly oscillating phase contributions. Moreover, based on a semiclassical investigation at small scattering angles, it has been argued by Taulbjerg et al (1990) that a correct incorporation of the channel distortion by the second collision partner makes the SPB results agree rather closely with the corresponding ones in the IA. On the other hand, an improvement in the peaking approximations is required in order to obtain better agreement with the experimental (off-resonance) capture data at large scattering angles (Scheurer et al 1985, Baker et al 1988).

The paper is organized as follows. In section 2, the quantum mechanical formulation of the SPB theory is briefly discussed, and in section 3, the nuclear two-channel model is presented. In the following section, the two-channel impulse approximation is derived. The evaluation of the capture probability is described in sections 5 and 6 , and the transverse peaking approximation is discussed in section 7 . Numerical details of the calculation are given in section 8 . In section 9 , the transverse peaked iA is applied to electron capture in the reaction ${ }^{19} \mathrm{~F}(\mathrm{p}, \alpha){ }^{16} \mathrm{O}$ where first measurements have been made (Horsdal Pedersen 1987). Predictions for electron capture in the reactions ${ }^{12} \mathrm{C}(\mathrm{d}, \mathrm{p}){ }^{13} \mathrm{C},{ }^{32} \mathrm{~S}\left(\mathrm{p}, \mathrm{p}^{\prime}\right){ }^{32} \mathrm{~S}$ and ${ }^{16} \mathrm{O}(\mathrm{n}, \alpha){ }^{13} \mathrm{C}$ which have a different energy transfer to width ratio and different charge and velocity ratios in the incoming and outgoing channel, are also given. All calculations are restricted to capture from the $\mathrm{K}$ shell. The conclusion is drawn in the last section. Atomic units $(\hbar=m=e=1)$ are used unless otherwise indicated.

\section{Transition amplitude in the strong potential Born approximation}

For the sake of transparency, the collision is described in terms of a three-body problem, consisting of the projectile nucleus, the target nucleus and the active target electron. 
The spectator electrons may readily be included through the use of screened electronic potentials. The Hamiltonian of this system is given by

$$
H=H_{\text {int }}+T_{\mathrm{N}}+V_{\mathrm{N}}+T_{\mathrm{e}}+V_{\mathrm{P}}+V_{\mathrm{T}} \text {. }
$$

$H_{\text {int }}$ describes the internal state of the projectile and target nucleus, while the internuclear motion is governed by the kinetic energy $T_{\mathrm{N}}$ and the potential $V_{\mathrm{N}} . T_{e}$ is the kinetic energy of the electron, $V_{\mathrm{P}}$ or $V_{\mathrm{T}}$ are the interactions between the electron and the projectile or target nucleus, respectively. For nuclear rearrangement collisions, the interactions in (2.1) are different in the initial channel, which will be denoted by an index $a$, and in the final channel, denoted by $b$. The electronic potentials are only uniquely defined outside the interaction region $R_{N}$ of the nuclei where nucleon transfer has ceased to take place. Formally, one may write

$$
V_{\mathrm{P}}=\sum_{\lambda} V_{\mathrm{P} \lambda} \hat{P}_{\lambda} \quad V_{\mathrm{T}}=\sum_{\lambda} V_{\mathrm{T} \lambda} \hat{P}_{\lambda}
$$

where $\hat{P}_{\lambda}$ is the projection operator onto channel $\lambda$, and $\lambda=a, b$. If we take the initial state $\tilde{\phi}_{i}^{\varphi}$ to describe an electron bound to the target, and a free internuclear motion, i.e. $\tilde{\phi}_{i}^{\varphi}$ as eigenstate to $H_{\mathrm{int}}^{(a)}+T_{\mathrm{N}}^{(a)}+T_{\mathrm{e} a}+V_{\mathrm{T} a}$, the transition amplitude for electron capture occurring during reactive nuclear scattering from channel $a$ to channel $b$ is obtained from

$$
\boldsymbol{W}_{\hat{j}}^{b a}=\left\langle\Psi_{j}^{\varphi(-)}\left|V_{\mathrm{N}}+\boldsymbol{V}_{\mathrm{P}}\right| \tilde{\phi}_{j}^{\varphi}\right\rangle
$$

where $\Psi_{f}^{\varphi(-)}$ is the exact scattering state to $H$, evolving asymptotically into a state $\tilde{\phi}_{f}^{\varphi}$ where the electron has been transferred to the projectile. Correspondingly, $\tilde{\phi}_{f}^{\varphi}$ is eigenstate to $H_{\mathrm{int}}^{(b)}+T_{\mathrm{N}}^{(b)}+T_{e b}+V_{\mathrm{P} b}$.

For energetic collisions with an impact velocity exceeding the classical orbiting velocity of the electron in the initial state, a perturbative approximation to $W_{f i}^{b a}$ is meaningful. For asymmetric collision systems where the projectile nuclear charge $\boldsymbol{Z}_{\mathrm{p}}$ is much smaller than the target nuclear charge $Z_{\mathrm{T}}$, an expansion of the exact scattering state in terms of $V_{\mathrm{P}}$ can be made. In the Lippmann-Schwinger equation for $\Psi_{f}^{\varphi(-)}$, the full propagator $G^{(-)}=(E-H-i \varepsilon)^{-1}$ is expanded in terms of $G_{\mathrm{NT}}^{(-)}=$ [E-(H-V $\left.\left.V_{\mathrm{P}}\right)-\mathrm{j} \varepsilon\right]^{-1}$, hence all potentials in $H$ except $V_{\mathrm{P}}$ are treated exactly. This leads to the following approximation for $\Psi_{f}^{\varphi(-)}$, which is correct to first order in $V_{\mathrm{P}}$

$$
\begin{aligned}
& \left|\Psi_{f}^{\varphi(-)}\right\rangle=\left|\tilde{\psi}_{f}^{(-) \mathrm{SPB}}\right\rangle+G_{\mathrm{NT}}^{(-)} V_{\mathrm{P}} G_{\mathrm{NT}}^{(-)} V_{\mathrm{T}}\left|\tilde{\psi}_{f}^{\varphi(-)}\right\rangle+\mathrm{O}\left(V_{\mathrm{P}}\right)^{2} \\
& \left|\tilde{\psi}_{f}^{(-) \mathrm{SPB}}\right\rangle=\left(1+G_{\mathrm{NT}}^{(-)} V_{\mathrm{T}}\right)\left|\tilde{\psi}_{f}^{\varphi(-)}\right\rangle
\end{aligned}
$$

where the strong potential Born wavefunction $\tilde{\psi}_{f}^{(-) \text {SPB }}$, which is the zeroth order approximation to $\Psi_{f^{(-)}}^{\varphi}$, has been introduced. The function $\tilde{\psi}_{f}^{\varphi(-)}$ which develops asymptotically into $\tilde{\phi}_{f}^{\varphi}$, describes an electron bound to the projectile, but with the internuclear potential included, i.e. $\tilde{\psi}_{f}^{\varphi(-)}$ is eigenstate to $H-V_{\mathrm{T}}$.

When (2.4) is inserted into the transition amplitude, a two-potential-like formula is obtained in first order in $V_{\mathrm{P}}$

$$
\begin{aligned}
& W_{f i}^{b a}=W_{f i}^{(0)}+W_{f i}^{(1)}-W_{f i}^{(01)}+O\left(V_{\mathrm{P}}\right)^{2} \\
& W_{f i}^{(0)}=\left\langle\tilde{\psi}_{f}^{(-) \mathrm{SPB}}\left|V_{N}\right| \tilde{\phi}_{i}^{\varphi}\right\rangle \quad W_{f i}^{(1)}=\left\langle\tilde{\psi}_{f}^{(-) \mathrm{SPB}}\left|V_{\mathrm{P}}\right| \tilde{\psi}_{i}^{\varphi(+)}\right\rangle \\
& W_{f i}^{(01)}=\left\langle\tilde{\psi}_{f}^{\varphi(-)}\left|V_{\mathrm{P}}\right| \tilde{\psi}_{i}^{\varphi(+)}-\tilde{\phi}_{i}^{\varphi}\right\rangle .
\end{aligned}
$$

The zeroth-order term in $V_{\mathrm{P}}, W_{f i}^{(0)}$, is the recoil term where electron capture is mediated by the internuclear potential $V_{\mathrm{N}}$. The first-order term $W_{f i}^{(1)}$ is the usual potential term (Jakubassa-Amundsen and Amundsen 1985, hereafter referred to as JAA). Here $\tilde{\psi}_{i}^{\varphi(t)}$, eigenstate to $H-V_{\mathrm{P}}$, has been introduced. In contrast to direct (i.e. non-rearrangement) electronic processes, there is an additional term present, the surface term $W_{f}^{(01)}$, which 
is of first order in $V_{\mathrm{p}}$, but in contrast to $W_{f i}^{(0)}$ or $W_{f i}^{(1)}$ of zeroth order in $V_{\mathrm{T}}$. It is important to include the surface term in the transition amplitude (although it has no correspondence in the semiclassical theory for large-angle electron capture as formulated by Amundsen and Jakubassa-Amundsen (1984b)) in order to reproduce the experimental dependence of the capture probability on the scattering angle in the absence of a nuclear resonance (Jakubassa-Amundsen 1987).

\section{Nuclear two-channel model}

The two-channel model for nuclear reactions (Amundsen 1986) relies on the assumption that only two channels are considered for the decay of the transiently formed compound nucleus, the elastic channel $a$ (i.e. the initial channel) and one inelastic channel $b$ (the final one when a reaction has taken place). If there are more open channeis available, this model is nevertheless applicable provided that couplings between these channels after the nuclear reaction can be neglected; consideration of the corresponding partial decay widths provides the correct weight factor for each channel.

Let $\psi_{K}(R)$ be a nuclear eigenfunction to $H_{\text {int }}+T_{N}+V_{N}$ where $R$ is the internuclear coordinate and $\boldsymbol{K}$ the asymptotic internuclear momentum. This function can be decomposed into two parts, the asymptotic function $\psi_{K}^{\infty}(\boldsymbol{R})$ which describes $\psi_{K}(\boldsymbol{R})$ far outside the nuclear interaction region, $R \gg R_{\mathrm{N}}$, plus a remainder $\psi_{K}^{\text {in }}(\boldsymbol{R})$ which basically describes $\psi_{\boldsymbol{K}}(\boldsymbol{R})$ inside the interaction region

$$
\psi_{\lambda \boldsymbol{K}}^{( \pm)}(\boldsymbol{R})=\psi_{\lambda \boldsymbol{K}}^{( \pm) i n}(\boldsymbol{R})+\psi_{\lambda \boldsymbol{K}}^{( \pm) \infty}(\boldsymbol{R})
$$

where the channel index $\lambda(\lambda=a, b)$ has been included. The inner wavefunction $\psi_{K}^{\text {in }}$ will not be needed explicitly in the following. The asymptotic functions are

$$
\begin{gathered}
\psi_{a K}^{(+) \infty}(\boldsymbol{R})=(2 \pi)^{-3 / 2}\left\{\left[\mathrm{e}^{\mathrm{i} K_{a} R_{a}}+A_{a a}^{(+)}\left(K_{a}, \theta_{K, R_{a}}\right) \mathrm{e}^{\left.\mathrm{i} K_{a} R_{a} / R_{a}\right] \phi_{a}}\right.\right. \\
+A_{b a}^{(+)}\left(K_{a}, \theta_{K, R_{b}}\right)\left(\mathrm{e}^{\left.\left.\mathrm{i} K_{b} R_{b} / R_{b}\right) \phi_{b}\right\}}\right. \\
\psi_{b K^{\prime}}^{(-) \infty}(\boldsymbol{R})=(2 \pi)^{-3 / 2}\left\{\left[\mathrm{e}^{\mathrm{i} K_{b}^{\prime} R_{b}}+A_{b b}^{(-)}\left(K_{b}^{\prime}, \theta_{K^{\prime}, \boldsymbol{R}_{b}}\right) \mathrm{e}^{-\mathrm{i} K_{b}^{\prime} R_{b}} / R_{b}\right] \phi_{b}\right. \\
+A_{a b}^{(-)}\left(K_{b}^{\prime}, \theta_{\boldsymbol{K}^{\prime}, \boldsymbol{R}_{a}}\right)\left(\mathrm{e}^{\left.\left.-\mathrm{i} K_{a}^{\prime} \boldsymbol{R}_{a} / R_{b}\right) \phi_{a}\right\} .}\right.
\end{gathered}
$$

The function $\psi_{a K}^{(+)}$describes an incoming wave in channel $a$, while $\psi_{b K^{\prime}}^{(-) \infty}$ accounts for an outgoing wave in channel $b$. The wavefunctions $\phi_{a}$ and $\phi_{b}$ are channel eigenfunctions to $H_{\text {int }}$ and denote the internal state of the two collision partners. In the two-channel model, $\phi_{a}$ and $\phi_{b}$ are assumed to form a complete set of mutually orthogonal functions. The elastic scattering amplitude in channel $\lambda$ (including the Coulomb scattering amplitude) is termed $A_{\lambda \lambda}$, while $A_{b a}$ and $A_{a b}$ are the reaction amplitudes. If we restrict ourselves to isolated resonances which are of Breit-W̄igner type, and if nuclear background phases can be neglected, the reaction amplitude has the form (Lane and Thomas 1958, Taylor 1972)

$$
\begin{aligned}
& A_{b a}^{(+)}\left(K_{a}, \theta_{K_{a}, R_{b}}\right) \\
&=- \frac{\sqrt{\pi}}{K_{a}} \sum_{l^{\prime} J} \sum_{s s^{\prime}} \sqrt{2 l+1} \mathrm{e}^{\mathrm{i} \sigma_{a, l^{\prime}}+\mathrm{i} \sigma_{b, r^{\prime}}} \frac{\left(\Gamma_{a} \Gamma_{b}\right)^{1 / 2}}{E_{a}-E_{R}^{(a)}+\mathrm{i} \Gamma / 2} \\
& \times \sum_{m_{M}^{\prime} M} \sum_{m_{s} m_{s}^{\prime}}\left(s^{\prime} m_{s}^{\prime} l m_{l^{\prime}} \mid J M\right)\left(s m_{s} l 0 \mid J M\right)\left(s_{\mathrm{P}} m_{s_{\mathrm{P}}} s_{\mathrm{T}} m_{s_{\mathrm{T}}} \mid s m_{s}\right) \\
& \times\left(s_{\mathrm{P}}^{\prime} m_{s_{\mathrm{p}}}^{\prime} s_{\mathrm{T}}^{\prime} m_{s \uparrow}^{\prime} \mid s^{\prime} m_{s}^{\prime}\right) Y_{l^{\prime} m_{i}}(\Omega) .
\end{aligned}
$$

$\boldsymbol{K}_{\lambda}$ is the internuclear momentum and $\boldsymbol{R}_{\lambda}$ the internuclear coordinate in channel $\lambda, E_{a}$ the centre-of-mass (CM) energy in channel $a$, and $\Omega$ the solid angle of $\boldsymbol{R}_{b}$ with respect 
to $\boldsymbol{K}_{a}$ as quantization axis. For unpolarized particles, only the dependence on the polar angle $\theta_{\boldsymbol{K}_{a}, \boldsymbol{R}_{b}}$ survives in the transition probability, while the dependence on the azimuthal angle $\varphi$ drops out. Hence, a possible $\varphi$-dependence of the reaction amplitude will in the following be disregarded. In equation (3.3), it is assumed that the projectile and target nuclei have intrinsic spins $s_{\mathrm{p}}$ and $s_{\mathrm{T}}$, respectively (unprimed quantum numbers refer to channel $a$, primed ones to channel $b$ ), which couple to the channel spin $s$. In turn, $s$ is coupled with the angular momentum $l$ of the projectile to the total angular momentum $\bar{J}$ of the resonant state in the compound nucleus. The symbols in brackets are Clebsch-Gordan coefficients, $Y_{I^{\prime} m_{i}}$ is a spherical harmonic, and the sum runs over the corresponding magnetic quantum numbers, with an explicit dependence of $\boldsymbol{A}_{b a}^{(+)}$ on $m_{s_{\mathrm{P}}}, m_{s_{\mathrm{P}}}^{\prime}, m_{\mathrm{s}}$ and $m_{s_{\mathrm{T}}}^{\prime}$ left. $E_{R}^{(a)}$ is the $\mathrm{CM}$ resonance energy, $\Gamma$ the total $\mathrm{CM}$ width and $\Gamma_{\lambda}$ the partial $\mathrm{CM}$ width for channel $\lambda$. The phase $\sigma_{\lambda, l}=\arg \Gamma\left(l+1+\mathrm{i} \eta_{\lambda}\right)$ with $\eta_{\lambda}=Z_{\mathrm{P}_{\lambda}} Z_{\mathrm{T} \lambda} \mu_{\lambda} / K_{\lambda}$ and $\mu_{\lambda}=M_{\mathrm{P} \lambda} M_{\mathrm{T} \lambda} /\left(M_{\mathrm{P} \lambda}+M_{\mathrm{T} \lambda}\right)$ the reduced mass in channel $\lambda\left(M_{\mathrm{P} \lambda}\right.$ and $M_{\mathrm{T} \lambda}$ are the projecctile and target masses, respectively, in channel $\lambda$ ) is the Coulomb phase.

The following interrelations hold for the reaction amplitudes (Joachain 1983)

$$
\begin{aligned}
& A_{b a}^{(-)}\left(K_{a}, \theta_{K, R}\right)=A_{b a}^{(+)}\left(K_{a}, \theta_{-K, R}\right) \\
& K_{a} A_{b a}^{(+)}\left(K_{a}, \theta_{K, R}\right)=K_{b} A_{a b}^{(+)}\left(K_{b}, \theta_{K, R}\right) \\
& E_{K_{b}} \equiv \frac{\bar{K}_{b}^{2}}{2 \mu_{b}}=\frac{\bar{K}_{a}^{2}}{2 \mu_{a}}+Q \quad \hat{\boldsymbol{K}}_{b}=\hat{\boldsymbol{K}}_{a} \equiv \hat{\boldsymbol{K}}
\end{aligned}
$$

where $Q$ denotes the energy gain (or $Q$-value) of the reaction, i.e. the difference of the intrinsic nuclear energies in channel $a$ and $b, Q=E_{\mathrm{int}}^{a}-E_{\mathrm{int}}^{b}$, and $\hat{\boldsymbol{K}}$ is the direction of $\boldsymbol{K}$.

Strictly speaking, equation (3.2) for the asymptotic nuclear functions hold only for short-range internuclear potentials, whereas for Coulombic potentials, additional logarithmic phases appear (Lane and Thomas 1958). For fast collisions where the semiclassical straight-line approximation is valid, inclusion of these phases gives only insignificant modifications of the transition probability (Amundsen and Aashamar 1986). In the present case of charge transfer during nuclear reactions test calculations gave modifications of about one per cent. The reason for this small effect is that only phase differences in the same channel enter into the transition amplitude, which are of the order of the electronic energy transfer with respect to the collision energy.

\section{The impulse approximation in the two-channel model}

As the next step, the wavefunctions entering into the transition amplitude (2.5) have to be specified. $\tilde{\psi}_{i}^{\varphi(+)}$, which is eigenstate to $H_{\mathrm{int}}+T_{\mathrm{N}}+V_{\mathrm{N}}+T_{\mathrm{e}}+V_{\mathrm{T}}$ can approximately be represented as a product of a nuclear function $\psi_{\lambda K}$, the bound electronic target state $\varphi_{i}$, and a translational factor relating to the reference frame where $\varphi_{i}$ is defined (the target frame; Végh 1983). A similar representation holds for $\tilde{\psi}_{f}^{\varphi(-)}$, with the translational factor relating to the projectile frame. For the initial state $\tilde{\phi}_{i}^{\varphi}$, the nuclear function is replaced by a plane wave. Explicitiy,

$$
\begin{aligned}
& \left|\tilde{\phi}_{j}^{\varphi}\right\rangle=(2 \pi)^{-3 / 2}\left|\mathrm{e}^{\mathrm{i} K_{i a} R_{a}} \phi_{a} \mathrm{e}^{-\mathrm{i} \beta_{a} K_{i a} r_{T}} \varphi_{i}\left(\boldsymbol{r}_{\mathrm{T}}\right)\right\rangle \\
& \left|\tilde{\psi}_{i}^{(+)}\right\rangle=\left(1+G_{\mathrm{NT}}^{(+)} V_{\mathrm{N}}\right)\left|\tilde{\phi}_{i}^{\varphi}\right\rangle=\left|\psi_{a K_{i}}^{(+)} \mathrm{e}^{-\mathrm{i} \beta_{\mathrm{a}} \boldsymbol{K}_{i a} \boldsymbol{r}_{\mathrm{T}}} \boldsymbol{\varphi}_{i}\left(\boldsymbol{r}_{\mathrm{T}}\right)\right\rangle \\
& \left|\tilde{\psi}_{f}^{\varphi(-)}\right\rangle=\left|\psi_{b \boldsymbol{K}_{f}}^{(-)} \mathrm{e}^{\mathrm{i} \boldsymbol{\alpha}_{b} \boldsymbol{K}_{f^{\prime}} \mathbf{P}} \varphi_{f}\left(\boldsymbol{r}_{\mathrm{P}}\right)\right\rangle
\end{aligned}
$$


where $\alpha_{\lambda}=m /\left(m+M_{\mathrm{P}_{\lambda}}\right), \beta_{\lambda}=m /\left(m+M_{\mathrm{T} \lambda}\right), m$ the electron mass and $\varphi_{f}$ the bound electronic projectile state. In using the notation $\boldsymbol{r}_{\mathrm{T}}$ and $\boldsymbol{r}_{\mathrm{P}}$ for the electronic coordinate with respect to the target and projectile, respectively, the assumption $r=r_{a} \approx r_{b}$ has been made, i.e. channel-specific differences in the electronic coordinate are neglected.

In order to write the SPB wavefunction $\tilde{\psi}_{f}^{(-) \mathrm{SPB}}$ from (2.4) in a more convenient way, use is made of the fact that the operator $\left(1+G_{\mathrm{NT}}^{(-)} V_{\mathrm{T}}\right)$ acting on an eigenstate to $H_{\mathrm{int}}+T_{\mathrm{N}}+V_{\mathrm{N}}+T_{\mathrm{e}}$ leads to an electronic off-shell wavefunction $\psi_{\mathrm{q}, \omega}$ (JAA). Inserting a complete set of such eigenstates into the expression for $\tilde{\psi}_{f}^{(-) \mathrm{SPB}}$ and making use of the completeness of the two-channel functions, one obtains

$$
\begin{aligned}
\left|\tilde{\psi}_{f}^{(-) S P B}\right\rangle=\int & \mathrm{d} \boldsymbol{K} \mathrm{d} \boldsymbol{q}\left\{\left|\psi_{a \boldsymbol{K}}^{(+)} \mathrm{e}^{-\mathrm{i} \beta \boldsymbol{K} \boldsymbol{r}_{\mathrm{T}}} \psi_{\boldsymbol{q}, \omega}\left(\boldsymbol{r}_{\mathrm{T}}\right)\right\rangle\left\langle\psi_{a \boldsymbol{K}}^{(+)} \mathrm{e}^{-\mathrm{i} \beta \boldsymbol{k} \boldsymbol{r}_{\mathrm{T}}} \boldsymbol{q}\left(\boldsymbol{r}_{\mathrm{T}}\right)\right|\right. \\
& \left.+\left|\psi_{b \boldsymbol{K}}^{(-)} \mathrm{e}^{-\mathrm{i} \beta \boldsymbol{K} \boldsymbol{r}_{\mathrm{T}}} \psi_{\boldsymbol{q}, \omega}\left(\boldsymbol{r}_{\mathrm{T}}\right)\right\rangle\left\langle\psi_{b \boldsymbol{K}}^{(-)} \mathrm{e}^{-\mathrm{i} \beta \boldsymbol{K} \boldsymbol{r}_{\mathrm{T}}} \boldsymbol{q}\left(\boldsymbol{r}_{\mathrm{T}}\right)\right|\right\}\left|\tilde{\psi}_{f}^{\varphi(-)}\right\rangle
\end{aligned}
$$

with $\omega=E-E_{\mathrm{im}}-K^{2} / 2 \mu$ where $E$ is the total energy, and $\boldsymbol{q}\left(\boldsymbol{r}_{\mathrm{T}}\right)$ denotes an electronic plane wave of momentum $q$ in the target reference frame. In the following, the on-shell approximation is made, i.e. the electronic function $\psi_{q, \omega}$ is replaced by a target continuum eigenstate with momentum $\boldsymbol{q}, \varphi_{\boldsymbol{q}}\left(\boldsymbol{r}_{\mathrm{T}}\right)$. In this 'impulse' approximation, the transition amplitude (2.5) reduces to

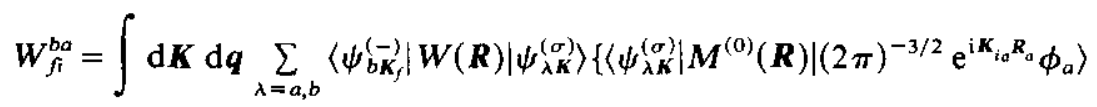

$$
\begin{aligned}
& \left.+\left\langle\psi_{\lambda K}^{(\sigma)}\left|M^{(1)}(\boldsymbol{R})\right| \psi_{a K_{i}}^{(+)}\right\rangle\right\}-\left\langle\psi_{b K_{f}}^{(-)}\left|S^{(1)}(\boldsymbol{R})\right| \psi_{a K_{i}}^{(+)}-(2 \pi)^{-3 / 2} \mathrm{e}^{\mathrm{i} \boldsymbol{K}_{i a} \boldsymbol{R}_{a}} \phi_{a}\right\rangle \\
& M^{(0)}(\boldsymbol{R}) \equiv\left\langle\mathrm{e}^{-\mathrm{i} \beta \boldsymbol{K}_{\mathrm{T}}} \varphi_{\boldsymbol{q}}\left(\boldsymbol{r}_{\mathrm{T}}\right)\left|V_{\mathrm{N}}\right| \mathrm{e}^{-\mathrm{i} \beta_{a} \boldsymbol{K}_{i a} \boldsymbol{r}_{\mathrm{T}}} \varphi_{i}\left(\boldsymbol{r}_{\mathrm{T}}\right)\right\rangle \\
& \boldsymbol{M}^{(1)}(\boldsymbol{R}) \equiv\left\langle\mathrm{e}^{-\mathrm{i} \beta \boldsymbol{K}_{\mathrm{T}}} \boldsymbol{\varphi}_{q}\left(\boldsymbol{r}_{\mathrm{T}}\right)\left|V_{\mathrm{P}}\right| \mathrm{e}^{-\mathrm{i} \beta_{a} \boldsymbol{K}_{i a} \boldsymbol{r}_{\mathrm{T}}} \varphi_{i}\left(\boldsymbol{r}_{\mathrm{T}}\right)\right\rangle \\
& W(\boldsymbol{R}) \equiv\left\langle\mathrm{e}^{\mathrm{i} \alpha_{b} \boldsymbol{K}_{f b} \boldsymbol{r}_{\mathrm{P}}} \varphi_{f}\left(\boldsymbol{r}_{\mathrm{P}}\right) \mid \mathrm{e}^{-\mathrm{i} \beta \boldsymbol{K}_{\boldsymbol{T}_{\mathrm{T}}}} \boldsymbol{q}\left(\boldsymbol{r}_{\mathrm{T}}\right)\right\rangle \\
& S^{(1)}(R) \equiv\left\langle\mathrm{e}^{\mathrm{i} \alpha_{b} \kappa_{f b} r_{\mathrm{P}}} \varphi_{f}\left(\boldsymbol{r}_{\mathrm{P}}\right)\left|\mathrm{V}_{\mathrm{P}}\right| \mathrm{e}^{-\mathrm{i} \beta_{a} K_{i a} \boldsymbol{r}_{\mathrm{T}}} \varphi_{i}\left(\boldsymbol{r}_{\mathrm{T}}\right)\right\rangle \text {. }
\end{aligned}
$$

The parameter $\sigma$ has to be taken $\sigma=+$ for $\lambda=a$ and $\sigma=-$ for $\lambda=b$. The electronic matrix elements describing potential and recoil ionization to an intermediate target continuum state are denoted by $M^{(1)}(R)$ and $M^{(0)}(R)$, respectively. The subsequent capture to a projectile state is mediated by the overlap $W(R) . S^{(1)}(R)$ is the first-order transfer matrix element from the surface term.

\section{Separation of atomic and nuclear transitions}

The evaluation of the nuclear matrix elements in (4.3) is facilitated by the large difference in the length scales of the nuclear and the atomic wavefunctions. Typical electronic dimensions are the shell radius or the inverse minimum momentum transfer, while the nuclear dimension is given by the radius $R_{N}$ of the nucleus which is several orders of magnitude smaller. Hence, for $R<R_{\mathrm{N}}$, the electronic matrix elements $M^{(1)}(R), W(R)$ and $S^{(1)}(R)$ can be replaced by their values at $R=0$, while for $R>R_{\mathrm{N}}$, the nuclear wavefunctions can be approximated by their asymptotic values (3.2). When the decomposition (3.1) for the nuclear functions is used, the nuclear matrix elements are thus approximated in the following way

$$
\left\langle\psi_{\lambda K}^{(\sigma)}\left|M^{(1)}(\boldsymbol{R})\right| \psi_{a K_{i}}^{(+)}\right\rangle \approx\left\langle\psi_{\lambda K}^{(\sigma) \infty}\left|M^{(1)}(\boldsymbol{R})-M^{(1)}(0)\right| \psi_{a K_{i}}^{(+)}\right\rangle+\left\langle\psi_{\lambda K}^{(\sigma)}\left|M^{(1)}(0)\right| \psi_{a K_{i}}^{(+)}\right\rangle
$$


with a similar expression for the matrix element involving $W(R)$ and $S^{(1)}(R)$. The contributions to $W_{f i}^{b a}$ which are proportional to $M^{(1)}(0), S^{(1)}(0)$ or $W(0)$ are called sticking terms, since they describe electron transfer during nuclear contact.

The sticking contributions where the nuclear matrix elements are formed with the exact nuclear functions (like in the last term of (5.1)) deserve special consideration. We make the assumption that the electronic matrix elements $\left(M^{(1)}(0), W(0), S^{(1)}(0)\right)$ are independent of the nuclear functions such that the nuclear matrix elements become proportional to the overlap of the nuclear functions. If these functions pertain to the same channel, or if proton transfer is excluded, this approximation is exact. For the general case of an $a \rightarrow b$ or $b \rightarrow a$ nuclear transition the influence of the electronic matrix elements on the nuclear functions during nuclear contact is expected to be small compared with the effect of the electronic energy transfer on the reaction amplitudes. With this approximation, we have the following relations

$$
\begin{aligned}
& M_{a}(0) \equiv\left\langle\psi_{a K}^{(+)}\left|M^{(1)}(0)\right| \psi_{a K_{i}}^{(+)}\right\rangle=-Z_{\mathbf{P a}} M_{2 a}(\mathbf{q})\left\langle\psi_{a K}^{(+)} \mid \psi_{a K}^{(+)}\right\rangle \\
& M_{b}(0) \equiv\left\langle\psi_{b K}^{(-)}\left|M^{(1)}(0)\right| \psi_{a K_{i}}^{(+)}\right\rangle=-Z_{\mathbf{P b}} M_{2 b}(\boldsymbol{q})\left\langle\psi_{b K}^{(-)} \mid \psi_{a K_{i}}^{(+)}\right\rangle \\
& M_{2 \lambda}(\boldsymbol{q})=\left\langle\mathrm{e}^{-\mathrm{i} \beta_{\lambda} K_{\lambda} \boldsymbol{r}_{\mathrm{T}}} \varphi_{\boldsymbol{q}}^{(\lambda)}\left(\boldsymbol{r}_{\mathrm{T}}\right)\left|1 / \boldsymbol{r}_{\mathrm{T}}\right| \mathrm{e}^{-\mathrm{i} \beta_{a} K_{i a} \boldsymbol{r}_{\mathrm{T}}} \varphi_{i}\left(\boldsymbol{r}_{\mathrm{T}}\right)\right\rangle \\
& W_{a}(0) \equiv\left\langle\psi_{b \tilde{f}_{j}}^{(-)}|W(0)| \psi_{a K}^{(+)}\right\rangle=\varphi_{f}^{*}\left(\mathbf{q}-\alpha_{b} \boldsymbol{K}_{f b}-\beta_{a} K_{a}\right)\left\langle\psi_{b \tilde{K}_{j}}^{(-)} \mid \psi_{a K}^{(+)}\right\rangle \\
& W_{b}(0) \equiv\left\langle\psi_{b \boldsymbol{K}_{f}}^{(-)}|W(0)| \psi_{b \boldsymbol{K}}^{(-)}\right\rangle=\varphi_{f}^{*}\left(\boldsymbol{q}-\alpha_{b} \boldsymbol{K}_{f b}-\beta_{b} \boldsymbol{K}_{b}\right)\left\langle\psi_{b \tilde{K}_{f}}^{(-)} \mid \psi_{b \boldsymbol{K}}^{(-)}\right\rangle \\
& W^{(01)}(0) \equiv\left\langle\psi_{b \tilde{K}_{f}}^{(-)}\left|S^{(1)}(0)\right| \psi_{a K_{i}}^{(+)}\right\rangle=-Z_{\mathrm{Pa}} M_{5}\left\langle\psi_{b \hat{K}_{f}}^{(-)} \mid \psi_{a \mathbf{K}_{i}}^{(+)}\right\rangle \\
& M_{5}=\left\langle\varphi_{f}\left(\boldsymbol{r}_{\mathrm{T}}\right) \mathrm{e}^{\mathrm{i} \alpha_{\mathrm{b}} \boldsymbol{K}_{\mathrm{fb}} \boldsymbol{r}_{\mathrm{T}}}\left|1 / \boldsymbol{r}_{\mathrm{T}}\right| \mathrm{e}^{-\mathrm{i} \beta_{\mathrm{a}} \boldsymbol{K}_{\mathrm{ta}} \boldsymbol{r}_{\mathrm{T}}} \varphi_{i}\left(\boldsymbol{r}_{\mathrm{T}}\right)\right\rangle
\end{aligned}
$$

where $\varphi_{f}^{*}(\boldsymbol{k})$ is the Fourier transform of the bound electronic projectile state. In the matrix elements of $W(0)$ and $S^{(1)}(0)$, the reduced final momentum $\tilde{\boldsymbol{K}}_{f}=\boldsymbol{K}_{f}\left(1-\alpha_{b}\right)$ has to be used instead of $\boldsymbol{K}_{f}$, because the translational factor $\exp \left(\mathrm{i} \alpha_{b} \boldsymbol{K}_{f b} \boldsymbol{R}_{b}\right)$ is dropped at zero internuclear distance and hence the energy of the internuclear motion must not contain the translational energy $v_{b}^{2} / 2$ of the electron, where $v$ is the collision velocity (JAA). The overlap of the nuclear functions is calculated with the help of the relations (Joachain 1983)

$$
\begin{aligned}
\left\langle\psi_{\lambda \boldsymbol{K}}^{(\sigma)} \mid \psi_{\lambda \boldsymbol{K}^{\prime}}^{(\sigma)}\right\rangle & =\delta\left(\boldsymbol{K}_{\lambda}-\boldsymbol{K}_{\lambda}^{\prime}\right) \\
\left\langle\psi_{b \boldsymbol{K}}^{(-)} \mid \psi_{a K^{\prime}}^{(+)}\right\rangle & =-2 \pi \mathrm{i} \delta\left(E_{K_{b}}-E_{K_{a}^{\prime}}-Q\right)\left\langle\psi_{b \boldsymbol{K}}^{(-)}\left|V_{N}\right| \phi_{a}(2 \pi)^{-3 / 2} \mathrm{e}^{\left.\mathrm{i} \boldsymbol{K}_{a}^{\prime} \boldsymbol{R}_{a}\right\rangle}\right. \\
& =\frac{\mathrm{i}}{2 \pi \sqrt{\mu_{\bar{a}} \mu_{b}}} \sqrt{\frac{K_{a}^{\prime}}{K_{b}}} \delta\left(E_{K_{b}}-E_{K_{a}^{\prime}}-Q\right) A_{b a}^{(+)}\left(K_{a}^{\prime}, \theta_{K_{b}, \boldsymbol{K}_{a}^{\prime}}\right)
\end{aligned}
$$

where the definitions of the energies are given in (3.4).

\section{Evaluation of the transition amplitude}

The evaluation of the transition amplitude proceeds in a similar way as in the onechannel case (JAA). Only those terms which are linear in the reaction amplitude $A_{b a}$ will be retained: Since the channels $a$ and $b$ are different, the zeroth-order terms in $A_{b a}$ vanish, while the second- and higher-order terms describe multiple nuclear scattering and hence are much smaller than those relating to a single scattering. 
$W_{f i}^{b a}$ from (4.3) is decomposed in the following way

$$
W_{f i}^{b a}=W_{f i}^{(0)}+W_{A}^{(1)}+W_{\mathrm{B}}^{(1)}+W_{\mathrm{C}}^{(1)}+W_{\mathrm{S}}^{(1)}-W_{f i}^{(01)}
$$

The first contribution, $W_{f i}^{(0)}$, is the recoil term, which can be evaluated without an approximation of type (5.1). Due to the restriction to first-order terms in $A_{b a}$, the matrix element of $M^{(0)}(\boldsymbol{R})$ from (4.3) has only to be evaluated in channel $b$. Expanding $M^{(0)}(\boldsymbol{R})$ up to first order in the small parameter $\beta$, one obtains

$$
\begin{aligned}
& \boldsymbol{R}_{b} \equiv\left\langle\psi_{b \boldsymbol{K}}^{(-)}\left|M^{(0)}(\boldsymbol{R})\right|(2 \pi)^{-3 / 2} \mathrm{e}^{\mathrm{i} \boldsymbol{K}_{i a} \boldsymbol{R}_{a}} \phi_{a}\right\rangle \\
& \quad=\mathrm{i}\left\langle\psi_{b \boldsymbol{K}}^{(-)}\left|M_{3}(\boldsymbol{q}) \beta_{a} \boldsymbol{K}_{a} V_{N}-V_{N} M_{3}(\boldsymbol{q}) \beta_{a} \boldsymbol{K}_{i a}\right|(2 \pi)^{-3 / 2} \mathrm{e}^{\mathrm{i} \boldsymbol{K}_{i a} \boldsymbol{R}_{a}} \phi_{a}\right\rangle+\mathrm{O}\left(\beta^{2}\right) \\
& M_{3}(\boldsymbol{q})=\left\langle\varphi_{\boldsymbol{q}}\left(\boldsymbol{r}_{\mathrm{T}}\right)\left|\boldsymbol{r}_{\mathrm{T}}\right| \varphi_{i}\left(\boldsymbol{r}_{\mathrm{T}}\right)\right\rangle .
\end{aligned}
$$

Making use of the fact that on the energy shell, i.e. for $E_{K_{h}}=E_{K_{i n}}+Q$, one has the identity $\left\langle\psi_{b K}^{(-)}\left|V_{N}\right| \phi_{a} \exp \left(i K_{i a} R_{a}\right)\right\rangle=\left\langle\phi_{b} \exp \left(i K_{b} R_{b}\right)\left|V_{N}\right| \psi_{a K_{i}}^{(+)}\right\rangle$, the nuclear matrix element in (6.2) is symmetrized and expressed by means of (5.3) in terms of the reaction amplitude

$$
\begin{gathered}
R_{b}=-\frac{\mathrm{i}}{(2 \pi)^{2} \sqrt{\mu_{i a} \mu_{i b}}}\left[M_{3 b}(\boldsymbol{q}) \beta_{a} K_{a} A_{b a}^{(+)}\left(K_{i a}, \theta_{\boldsymbol{K}_{b}, \boldsymbol{K}_{i a}}\right) \sqrt{K_{i a} / K_{b}}\right. \\
\left.-M_{3 a}(\boldsymbol{q}) \beta_{a} \boldsymbol{K}_{i a} A_{b a}^{(+)}\left(K_{a}, \theta_{\boldsymbol{K}_{b}, \boldsymbol{K}_{i a}}\right) \sqrt{K_{a} / K_{i b}}\right]
\end{gathered}
$$

where $\mu_{i \lambda}$ is the channel-specific reduced mass of the projectile and the target nucleus plus electron, $\mu_{i \lambda}=M_{\mathrm{P}_{\lambda}}\left(M_{\mathrm{T \lambda}}+m\right) /\left(M_{\mathrm{P \lambda}}+M_{\mathrm{T \lambda}}+m\right)$, and $M_{3 \lambda}(q)$ results from $M_{3}(q)$ defined in (6.2) with $\varphi_{q}\left(\boldsymbol{r}_{\mathrm{T}}\right)$ replaced by $\varphi_{q}^{(\lambda)}\left(\boldsymbol{r}_{\mathrm{T}}\right)$. The on-shell approximation implied in the step from (6.2) to (6.3) holds to the same accuracy as in the one-channel case (JAA), i.e. to the order of $\left(\Delta K_{\lambda} / K_{\lambda}\right)^{2}$ where $\Delta K_{\lambda}$ is the channel-specific momentum transferred to the electron.

Since the matrix element $R_{b}$ is already linear in $A_{b a}$, it has to be folded with the zeroth-order term from $\left\langle\psi_{b K_{f}}^{(-)}|W(R)| \psi_{b K}^{(-)}\right\rangle$in (4.3). This results in the recoil term

$$
\begin{aligned}
W_{f i}^{(0)}=-\frac{\mathrm{i}}{4 \pi^{2}} & \frac{1}{\sqrt{\mu_{i a} \mu_{i b}}} \int \mathrm{d} q \varphi_{f}^{*}\left(q-\alpha_{b} K_{f b}\right)\left(\beta_{a} M_{3 b}(q) K_{a} \frac{K_{f b}-q}{\left|K_{f b}-q\right|} A_{b a}^{(+)}\left(K_{i a}, \theta_{K_{i a}, K_{f b}-q}\right)\right. \\
& \left.\times \sqrt{\frac{K_{i a}}{\left|K_{f b}-q\right|}}-\beta_{a} M_{3 a}(q) K_{i a} \sqrt{\frac{K_{a}}{K_{i b}}} A_{b a}^{(+)}\left(K_{a}, \theta_{K_{i a}, K_{f b}-q}\right)\right)
\end{aligned}
$$

where $\boldsymbol{K}_{a}$ is the channel- $a$ momentum related to the channel- $b$ momentum $\boldsymbol{K}_{b}=\boldsymbol{K}_{f b}-\boldsymbol{q}$ according to (3.4). The contribution to $W_{j i}^{(0)}$ which is proportional to $A_{b a}^{(+)}\left(K_{i a}, \theta\right)$ describes electron transfer after the reaction has taken place, while the second term corresponds to ionization to an intermediate state in channel $a$, followed by the nuclear reaction and the capture in channel $b$. Here and in the following we use the name 'electron transfer' for the process including ionization and capture, while 'capture' means just the second part of this process.

All other contributions to $W_{f i}^{b a}$ are evaluated with the small parameter $\beta$ eventually set equal to zero. The terms $W_{A}^{(1)}$ and $W_{B}^{(1)}$ arise from the potential term $W_{f i}^{(1)}$ and comprise the transitions at large internuclear separation

$W_{\mathrm{A}}^{(1)}+W_{\mathrm{E}}^{(1)} \equiv \int \mathrm{d} \boldsymbol{K} \mathrm{d} q \sum_{\lambda=a, b}\left\langle\psi_{b K_{j}}^{(-)}|W(\boldsymbol{R})| \psi_{\lambda \boldsymbol{K}}^{(\sigma) \infty}\right\rangle\left\langle\psi_{\lambda \boldsymbol{K}}^{(\boldsymbol{\sigma}) \infty}\left|\boldsymbol{M}^{(1)}(\boldsymbol{R})\right| \psi_{a \boldsymbol{K}_{i}}^{(+) \infty}\right\rangle$. 
Both channels $(\lambda=a, b)$ contribute. The basic ingredients for the evaluation of these and the remaining terms of $W_{f i}^{b a}$ will be demonstrated for the matrix element of $M^{(1)}(\boldsymbol{R})$. First, the Fourier representation of the Coulomb potential entering into $M^{(1)}(R)$ is introduced, such that

$$
\begin{aligned}
& M_{b}^{\infty} \equiv\left\langle\psi_{b \boldsymbol{K}}^{(-) \infty}\left|M^{(1)}(\boldsymbol{R})\right| \psi_{a K_{i}}^{(+) \infty}\right\rangle \\
& =-\frac{1}{2 \pi^{2}}\left\langle\psi_{b K}^{(-) \infty}\left|Z_{\mathrm{P}} \int \frac{\mathrm{d} s}{s^{2}} \mathrm{e}^{-\mathrm{i} s R} M_{1}(q, s)\right| \psi_{a K_{i}}^{(+) \infty}\right\rangle \\
& M_{1}(\boldsymbol{q}, \boldsymbol{s})=\left\langle\mathrm{e}^{-\mathrm{i} \beta_{\lambda} K_{\lambda} r_{T}} \varphi_{\boldsymbol{q}}\left(\boldsymbol{r}_{\mathrm{T}}\right)\left|\mathrm{e}^{\mathrm{i} s \boldsymbol{r}_{\mathrm{T}}}\right| \mathrm{e}^{-\mathrm{i} \beta_{\mathrm{a}} \boldsymbol{K}_{i a} \boldsymbol{r}_{\mathrm{T}}} \varphi_{i}\left(\boldsymbol{r}_{\mathrm{T}}\right)\right\rangle .
\end{aligned}
$$

Making use of the fact that $\left\langle\phi_{a} \mid \phi_{b}\right\rangle=0$ and retaining only the linear terms in the reaction amplitude, one obtains

$$
\begin{aligned}
M_{b}^{\infty}=-\frac{Z_{\mathrm{P} b}}{2 \pi^{2}} \int \frac{\mathrm{d} s}{s^{2}} M_{1 b}(q, s) \frac{1}{(2 \pi)^{3}} \int \mathrm{d} \boldsymbol{R}_{b} \mathrm{e}^{-\mathrm{is} \boldsymbol{R}_{b}} \mathrm{e}^{-\mathrm{i} K_{b} \mathbf{R}_{b}} \frac{\mathrm{e}^{\mathrm{i} K_{i b} R_{b}}}{R_{b}} A_{b a}^{(+)}\left(K_{i a}, \theta_{K_{i a}, R_{b}}\right) \\
-\frac{Z_{\mathrm{P} a}}{2 \pi^{2}} \int \frac{\mathrm{d} s}{s^{2}} M_{1 a}(q, s) \frac{1}{(2 \pi)^{3}} \int \mathrm{d} \boldsymbol{R}_{a} \mathrm{e}^{-\mathrm{i} s \boldsymbol{R}_{a}} \mathrm{e}^{\mathrm{i} K_{i a} \boldsymbol{R}_{a}} \frac{\mathrm{e}^{\mathrm{i} K_{a} R_{a}}}{R_{a}} A_{a b}^{(-)^{*}}\left(K_{b}, \theta_{K_{b}, R_{a}}\right)
\end{aligned}
$$

where $M_{1 \lambda}(q, s), \lambda=a, b$, is obtained from (6.6) by means of replacing $\varphi_{q}$ by $\varphi_{q}^{(\lambda)}$ which denotes a target eigenstate to the charge $Z_{\mathrm{T} \lambda}$. The integration over the internuclear coordinate in (6.7) is easily performed with the help of the relations, the first of which being valid for $R \rightarrow \infty$,

$$
\begin{aligned}
& \mathrm{e}^{ \pm \mathrm{i} K \boldsymbol{R}}= \pm \frac{2 \pi}{\mathrm{i} K R}\left[\mathrm{e}^{ \pm \mathrm{i} K R} \delta(\hat{\boldsymbol{R}}-\hat{\boldsymbol{K}})-\mathrm{e}^{\mp \mathrm{i} K R} \delta(\hat{\boldsymbol{R}}+\hat{\boldsymbol{K}})\right] \\
& \int_{0}^{\infty} \mathrm{d} R \mathrm{e}^{\mathrm{i} k \boldsymbol{R}}=\pi \delta(k)+\mathrm{P} \frac{\mathrm{i}}{k}
\end{aligned}
$$

where $P$ denotes the principal value. Disregarding the terms with strongly oscillating phase factors, i.e. keeping only these terms which contain differences of internuclear momenta, $M_{b}^{\infty}$ finally reduces to

$$
\begin{aligned}
M_{b}^{\infty}=-\frac{\mathrm{i} Z_{\mathrm{P} b}}{8 \pi^{4}} \int & \frac{\mathrm{d} s}{s^{2}} M_{1 b}(q, s) \frac{1}{\left|K_{b}+s\right|} A_{b a}^{(+)}\left(K_{i a}, \theta_{K_{i a}, K_{b}+s}\right) \\
& \times\left(\pi \delta\left(K_{i b}-\left|K_{b}+s\right|\right)+\frac{\mathrm{i}}{K_{i b}-\left|K_{b}+s\right|}\right) \\
& -\frac{\mathrm{i} Z_{\mathrm{P} a}}{8 \pi^{4}} \int \frac{\mathrm{d} s}{s^{2}} M_{1 a}(q, s) \frac{1}{\left|K_{i a}-s\right|} A_{a b}^{(-)^{*}}\left(K_{b}, \theta_{K_{a r}-K_{i a}+s}\right) \\
& \times\left(\pi \delta\left(K_{a}-\left|K_{i a}-s\right|\right)+\frac{\mathrm{i}}{K_{a}-\left|K_{i a}-s\right|}\right) .
\end{aligned}
$$

Proceeding in a similar way with the other matrix elements occurring in (6.5), the momentum integrals are easily carried out. Let us denote by $W_{A}^{(1)}$ that part of (6.5) which describes electron transfer before the nuclear reaction (a term proportional to 
$\left.A_{a b}^{(+)}\left(K_{f b}, \theta\right)\right)$ and electron transfer after the nuclear reaction (a term proportional to $\left.A_{b a}^{(+)}\left(K_{i a}, \theta\right)\right)$. Then we obtain

$$
\begin{aligned}
W_{\mathrm{A}}^{(1)}=\frac{1}{8 \pi^{4} \mathrm{i}} \int & \mathrm{d} q \varphi_{f}^{*}\left(q-\alpha_{b} K_{f b}\right) \int \frac{\mathrm{d} s}{s^{2}}\left[M_{1 a}(q, s) \frac{Z_{\mathrm{Pa}}}{\left|K_{i a}-s+q\right|} A_{a b}^{(+)}\left(K_{f b}, \theta_{K_{f b}, K_{i a}-s+q}\right)\right. \\
& \times\left(\pi \delta\left(\Delta K_{1}\right)+\frac{\mathrm{i}}{\Delta K_{1}}\right)+M_{1 b}(q, s) \frac{Z_{\mathrm{Pb}}}{\left|K_{f b}-q+s\right|} A_{b a}^{(+)}\left(K_{i a}, \theta_{K_{i a}, K_{f b}-q+s}\right) \\
& \left.\times\left(\pi \delta\left(\Delta K_{1}^{\prime}\right)+\frac{\mathrm{i}}{\Delta K_{1}^{\prime}}\right)\right]
\end{aligned}
$$

$W_{\mathrm{B}}^{(1)}$ collects the contributions which arise from ionization in channel $a$, but capture after the reaction in channel $b$

$$
\begin{aligned}
& W_{\mathrm{B}}^{(1)}=\frac{Z_{\mathrm{Pa}}}{8 \pi^{4} \mathrm{i}} \int \mathrm{d} \boldsymbol{q} \varphi_{f}^{*}\left(q-\alpha_{b} \boldsymbol{K}_{f b}\right) \int \frac{\mathrm{d} s}{s^{2}} M_{1 a}(q, s)\left[\frac{1}{\left|K_{f b}-q\right|} A_{b a}^{(+)}\left(\left|\boldsymbol{K}_{i a}-s\right|, \theta_{K_{i a}-s, K_{f b}-q}\right)\right. \\
& \times\left(\pi \delta\left(\Delta K_{2}^{\prime}\right)-\frac{\mathrm{i}}{\Delta K_{2}^{\prime}}\right)+\frac{1}{\left|K_{i a}-s\right|} A_{a b}^{(+)}\left(\left|K_{f b}-q\right|, \theta_{K_{f b}-q, K_{i a}-s}\right) \\
&\left.\times\left(\pi \delta\left(\Delta K_{2}\right)+\frac{\mathrm{j}}{\Delta K_{2}}\right)\right] \\
& \Delta K_{2}=K_{a}-\left|K_{i a}-s\right| \quad \Delta K_{2}^{\prime}=\left|K_{f b}-q\right|-K_{b}^{\prime}
\end{aligned}
$$

where $\boldsymbol{K}_{a}$ is defined below (6.4) and $\boldsymbol{K}_{b}^{\prime}$ is the channel-b momentum related to the channel- $a$ momentum $\boldsymbol{K}_{a}^{\prime}=\boldsymbol{K}_{i a}-s$ according to (3.4).

The next two contributions to $W_{f i}^{b a}$ originate from electron transfer while the two nuclei stick together. $W_{\mathrm{C}}^{(1)}$ in equation (6.1) results from ionization in channel $a$, but capture during nuclear contact. With $W_{a}(0)$ from $(5.2)$ one has

$$
\begin{aligned}
& W_{\mathrm{C}}^{(1)} \equiv \int \mathrm{d} \boldsymbol{K} \mathrm{d} \boldsymbol{q}\left[-\left\langle\psi_{b \tilde{\boldsymbol{K}}_{f}}^{(-) \infty}|W(0)| \psi_{a K}^{(+) \infty)}\right\rangle+W_{a}(0)\right]\left\langle\psi_{a \boldsymbol{K}}^{(+) \infty}\left|M^{(1)}(\boldsymbol{R})\right| \psi_{a K_{i}}^{(+) \infty}\right\rangle \\
&=\frac{Z_{\mathrm{Pa}}}{8 \pi^{4} \mathrm{i}} \int \mathrm{d} \boldsymbol{q} \varphi_{f}^{*}\left(q-\alpha_{b} \boldsymbol{K}_{f b}\right) \int \frac{\mathrm{d} s}{s^{2}} M_{1 a}(q, s)\left[\frac{1}{\tilde{K}_{f b}} A_{b a}^{(+)}\left(\left|\boldsymbol{K}_{i a}-s\right|, \theta_{\boldsymbol{K}_{i a}-s, K_{f b}}\right)\right. \\
& \times\left(-\pi \delta\left(\Delta K_{3}^{\prime}\right)+\frac{\mathrm{i}}{\Delta K_{3}^{\prime}}\right)-\frac{1}{\left|\boldsymbol{K}_{i a}-s\right|} A_{a b}^{(+)}\left(\tilde{K}_{f b}, \theta_{K_{f b}, \boldsymbol{K}_{i a}-s}\right)\left(\pi \delta\left(\Delta K_{3}\right)+\frac{\mathrm{i}}{\Delta K_{3}}\right) \\
&\left.+\frac{2 \pi}{\sqrt{\mu_{i a} \mu_{i b}}} \sqrt{\frac{\left|\boldsymbol{K}_{i a}-s\right|}{\tilde{K}_{f b}}} \delta\left(E_{\tilde{K}_{f b}}-E_{\left|K_{i a}-s\right|}-Q\right) A_{b a}^{(+)}\left(\left|K_{i a}-s\right|, \theta_{K_{f b}, K_{i a}-s}\right)\right]
\end{aligned}
$$

$$
\Delta K_{3}=\tilde{K}_{f a}-\left|K_{i a}-s\right| \quad \Delta K_{3}^{\prime}=\tilde{K}_{f b}-K_{b}^{\prime}
$$

where $M_{1 a}(q, s)$ is defined below (6.7). The corresponding channel-b contribution to $W_{C}^{(1)}$ is absent because it is of third order in the reaction amplitude.

The other sticking term, $W_{\mathrm{S}}^{(1)}$ in (6.1), results from ionization during nuclear contact, and capture in channel $b$. Only the contribution from channel $b$ has to be considered, 
and with $M_{b}(0)$ from (5.2) one finds

$$
\begin{aligned}
& \boldsymbol{W}_{\mathrm{S}}^{(1)} \equiv \int \mathrm{d} \boldsymbol{K} \mathrm{d} \boldsymbol{q}\left\langle\psi_{b K_{f}}^{(-) \infty}|W(\boldsymbol{R})| \psi_{b \boldsymbol{K}}^{(-) \infty}\right\rangle\left[-\left\langle\psi_{b \boldsymbol{K}}^{(-) \infty}\left|M^{(1)}(0)\right| \psi_{a K_{t}}^{(+) \infty}\right\rangle+M_{b}(0)\right] \\
& =-\frac{1}{4 \pi^{2} \mathrm{i}} \int \mathrm{d} \boldsymbol{q} \varphi_{f}^{*}\left(\boldsymbol{q}-\alpha_{b} \boldsymbol{K}_{f b}\right)\left[Z_{\mathrm{Pb}} M_{2 b}(\boldsymbol{q}) \frac{1}{\left|\boldsymbol{K}_{f b}-\boldsymbol{q}\right|} A_{b a}^{(+)}\left(K_{i a}, \theta_{\boldsymbol{K}_{a a}, K_{f h}-\boldsymbol{q}}\right)\right. \\
& \times\left(\pi \delta\left(\Delta K_{4}^{\prime}\right)+\frac{\mathrm{i}}{\Delta K_{4}^{\prime}}\right)+Z_{\mathbf{P a}} M_{2 a}(q) \frac{1}{K_{i a}} A_{a b}^{(+)}\left(\left|K_{f b}-q\right|, \theta_{\boldsymbol{K}_{f b}-\mathbf{q}, K_{i a}}\right) \\
& \times\left(\pi \delta\left(\Delta K_{4}\right)+\frac{\mathrm{i}}{\Delta K_{4}}\right)-2 \pi Z_{\mathrm{Pb}} M_{2 b}(q) \delta\left(E_{\left|K_{f b}-q\right|}-E_{K_{t b}}\right) \\
& \left.\times \frac{1}{\sqrt{\mu_{i a} \mu_{i b}}} A_{b a}^{(+)}\left(K_{i a}, \theta_{K_{f b}-\mathbf{q} . K_{i 2}}\right) \sqrt{\frac{K_{i a}}{\left|\boldsymbol{K}_{f b}-q\right|}}\right] \\
& \Delta K_{4}=K_{a}-K_{i a} \quad \Delta K_{4}^{\prime}=K_{i b}-\left|K_{f b}-q\right|
\end{aligned}
$$

with $M_{2 \lambda}(q)$ from (5.2) and $\boldsymbol{K}_{a}$ defined below equation (6.4). Both sticking contributions, $W_{\mathrm{C}}^{(1)}$ and $W_{\mathrm{S}}^{(1)}$, are basically proportional to the difference of the reaction amplitudes. For elastic scattering, i.e. when channel $b$ is formally set equal to channel $a$, these terms vanish in the absence of a nuclear resonance (JAA).

The last contribution to $W_{f i}^{b a}$ is the surface term which is composed of three parts

$$
\begin{gathered}
\boldsymbol{W}_{\hat{f}}^{(01)}=\left\langle\psi_{b \boldsymbol{K}_{f}}^{(-)}\left|S^{(1)}(\boldsymbol{R})\right| \psi_{a \boldsymbol{K}_{i}}^{(+) \infty}-(2 \pi)^{-3 / 2} \mathrm{e}^{\mathrm{i} \boldsymbol{K}_{i \mathrm{a}} \boldsymbol{R}_{a}} \phi_{a}\right\rangle-\left\langle\psi_{b \tilde{\boldsymbol{K}}_{f}}^{(-)}\left|S^{(1)}(0)\right| \psi_{a \boldsymbol{K}_{i}}^{(+) \infty}\right\rangle+W^{(01)}(0) \\
=W_{f i}^{\mathbf{R}(01)}+W_{f i}^{\mathrm{s}(01)}+W^{(01)}(0)
\end{gathered}
$$

$W_{f i}^{R(01)}$ is a recoil-type term resulting from transitions at large internuclear separation, while $W_{f i}^{S(01)}$ is the sticking contribution. A residual sticking term from the overlap of the nuclear wavefunction with a plane wave within the nuclear radius has been neglected as previously (Jakubassa-Amundsen $1987 \dagger$ ). The last term, $W^{(01)}(0)$, vanishes because the nuclear functions are off the energy shell (cf (5.2) and (5.3)). Evaluating the other terms, one obtains

$$
\begin{aligned}
& W_{f i}^{\mathrm{R}(01)}=-\frac{\mathrm{i}}{4 \pi^{2}} Z_{\overline{\mathrm{P} b}} \int \mathrm{dq} \frac{1}{\left|K_{f b}-q\right|} \varphi_{i}(\boldsymbol{q}) M_{4}(q) A_{b a}^{(+)}\left(K_{i a}, \theta_{\tilde{K}_{i a}, \tilde{K}_{f b}-q}\right)\left(\pi \delta\left(\Delta K_{4}^{\prime}\right)+\frac{\mathrm{i}}{\Delta K_{4}^{\prime}}\right) \\
& W_{f i}^{S(01)}=\frac{\mathrm{i}}{4 \pi^{2}} M_{S}\left[\frac{Z_{\mathrm{Pb}}}{\tilde{K}_{f b}} A_{b a}^{(+)}\left(K_{i b}, \theta_{K_{i a}, K_{f b}}\right)\left(\pi \delta\left(\Delta K_{S}^{\prime}\right)+\frac{\mathrm{i}}{\Delta K_{S}^{\prime}}\right)\right. \\
& \left.+\frac{Z_{\mathrm{Pa}}}{K_{i a}} A_{b a}^{(+)}\left(\tilde{\boldsymbol{K}}_{f a}, \theta_{\boldsymbol{K}_{f b}, \boldsymbol{K}_{i a}}\right) \frac{\tilde{\boldsymbol{K}}_{f a}}{\tilde{K}_{f b}}\left(\pi \delta\left(\Delta K_{5}\right)+\frac{\mathrm{i}}{\Delta K_{5}}\right)\right] \\
& M_{4}(\boldsymbol{q})=\left\langle\varphi_{f}\left(\boldsymbol{r}_{\mathrm{P}}\right) \mathrm{e}^{\mathrm{i} \omega_{b} \boldsymbol{K}_{f b} \boldsymbol{r}_{\mathrm{P}}}\left|1 / \boldsymbol{r}_{\mathrm{P}}\right| \mathrm{e}^{\left.\mathrm{i} \boldsymbol{q} \boldsymbol{r}_{\mathrm{P}}(2 \pi)^{-3 / 2}\right\rangle}\right. \\
& \Delta K_{5}^{\prime}=K_{i b}-\tilde{K}_{f b} \quad \Delta K_{5}=\tilde{K}_{f a}-K_{i a}
\end{aligned}
$$

where $\tilde{\boldsymbol{K}}_{f a}$ is the channel- $a$ momentum related to $\tilde{\boldsymbol{K}}_{f b}$ via (3.4), i.e. $\tilde{\boldsymbol{K}}_{f a}=\boldsymbol{K}_{a}\left(\boldsymbol{q}=\alpha_{b} \boldsymbol{K}_{f b}\right)$. The matrix element $M_{5}$ is defined in (5.2) with $\beta_{a}$ set equal to zero. The recoil surface term describes electron transfer in channel $b$, proceeding in a single step, while the sticking contribution describes electron transfer during nuclear contact and also vanishes in the absence of a nuclear resonance.

$\dagger$ In equation (11) of that paper, $\Delta E-v^{2} / 2+q v_{f}$ should read $\Delta E+v^{2} / 2$ in $W_{f i}^{S(0)}$, and in equation (9), $W_{\Omega}^{\langle(\mathbb{1})}$ should appear with a minus sign in $W_{f}^{\mathrm{SPB}}$. 
In the following, the nuclear momenta are expressed in terms of the collision velocity $v$, and differences of nuclear momenta are represented by the momentum transferred to the electron. To this aim, we make use of the fact that $m / M_{\mathrm{P}}, m / M_{\mathrm{T}}$ and $\Delta K / K$ are small quantities, such that in the momentum terms $\Delta K_{n}, \Delta K_{n}^{\prime}, n=1-5$ of equations (6.10)-(6.15) a linear expansion in the electronic momenta is sufficient. If no momentum differences are involved, the electronic momenta can be dropped whenever they are added to nuclear momenta. For the asymmetric collision systems considered here $\left(M_{\mathrm{P}} / M_{\mathrm{T}} \ll 1\right), \alpha \approx m / M_{\mathrm{P}}$ has to be retained, although $\beta \approx m / M_{\mathrm{T}}$ can be neglected (except in the recoil term). With the definitions $K_{i \lambda}=\mu_{i \lambda} v_{i \lambda}$ and $K_{f \lambda}=\mu_{f \lambda} v_{f \lambda}$, the following approximate relations hold

$$
\begin{aligned}
& \mu_{f \lambda} \approx \mu_{i \lambda}+1 \quad v_{i \lambda}-v_{f \lambda} \approx-\frac{1}{\mu_{i \lambda} v_{\lambda}}\left(\Delta E+\frac{v_{\lambda}^{2}}{2}\right) \\
& K_{f \lambda}-K_{i \lambda} \approx-\Delta E / v_{\lambda}+v_{\lambda} / 2 \\
& K_{a} \approx K_{i a}-\Delta E / v_{a}+v_{b}^{2} / 2 v_{a}-q v_{f b} / v_{a} \quad K_{b}^{\prime} \approx K_{i b}-v_{i a} s / v_{b} \\
& E_{\left|K_{f b}-q\right|}-E_{K_{i b}}=\left[\left(K_{f b}-q\right)^{2}-K_{i b}^{2}\right] /\left(2 \mu_{i b}\right) \approx-\Delta E+v_{b}^{2} / 2-q v_{f b} \\
& E_{\tilde{K}_{f b}}-E_{\left|K_{i a}-s\right|}-Q \approx-\Delta E-v_{b}^{2} / 2+s v_{i a}
\end{aligned}
$$

where $\Delta E=\varepsilon_{f b}-\varepsilon_{i a}$ denotes the difference between the energies of the bound electrons in the final and the initial state. The approximation $v_{\lambda} \equiv v_{i \lambda} \approx v_{f \lambda}$ is used for the collision velocity in channel $\lambda$, after the momentum differences have been carried out.

Collecting the above results, the transition amplitude for electron capture during reactive nuclear scattering can be written in the following form

$$
\begin{aligned}
& W_{f i}^{b a}=\frac{1}{4 \pi^{2} \mu_{b}}\left[A_{b a}^{(+)}\left(E_{f a}, \theta\right) a_{i i}^{(a)}+\int \mathrm{d} q a_{f q}^{(b)} A_{b a}^{(+)}\left(E_{q a}, \theta\right) a_{q i}^{(a)}+a_{f f}^{(b)} A_{b a}^{(+)}\left(E_{i a}, \theta\right)\right]+W_{f i}^{\mathrm{s}} \\
& E_{i a}=K_{i a}^{2} / 2 \mu_{i a} \quad E_{f a}=K_{f b}^{2} / 2 \mu_{f b}-v_{b}^{2} / 2-Q=E_{i a}-\left(\Delta E+v_{b}^{2} / 2\right) \\
& E_{q a}=K_{a}^{2} / 2 \mu_{i a}=E_{i a}-\Delta E+v_{b}^{2} / 2-q v_{f b} .
\end{aligned}
$$

Each term of $W_{f i}^{b a}$ factorizes into the electronic transition amplitudes $a^{(\lambda)}$ and the nuclear reaction amplitudes $A_{b a}^{(+)}$. The first term of $W_{f i}^{b a}$ describes electron transfer before the nuclear reaction, which takes place at an internuclear energy reduced by the energy transferred to the electron, the second term describes ionization in channel $a$, and the subsequent capture in channel $b$, integrated over the intermediate electronic states, while the third term describes electron transfer after the nuclear reaction. Correspondingly, $a_{i i}^{(a)}, a_{f q}^{(b)} a_{q i}^{(a)}$ and $a_{f f}^{(b)}$ denote the electronic amplitudes for electron transfer in channel $a$, ionization in channel $a$ and capture in channel $b$, and electron transfer in channel $b$, respectively. The last term in $(6.17), W_{f i}^{\mathrm{s}}$, comprises the sticking contributions resulting from either ionization $\left(W_{S}^{(1)}\right)$ or capture $\left(W_{C}^{(1)}\right)$ or transfer $\left(W_{f i}^{\text {S(01) }}\right)$ during nuclear contact.

\section{The transverse peaking approximation}

In order to evaluate the six-dimensional integrals $W_{\mathrm{A}}^{(1)}, W_{\mathrm{B}}^{(1)}$ and $W_{\mathrm{C}}^{(1)}$, a peaking approximation has to be introduced. This peaking approximation relies on the fact that for light projectiles, the momentum-space wavefunction $\varphi_{f}^{*}\left(q-\alpha_{b} K_{f b}\right)$ is strongly peaked for $q=\alpha_{b} K_{f b}$ while the remaining integrand varies slowly with $q$ in this region. 
Whereas in JAA the so-called full peaking approximation has been applied, where the momentum $q$ is replaced by $\alpha_{b} K_{f b}$ throughout in all matrix elements, we now use the less restrictive transverse peaking approximation. This approximation allows for a residual variation of $q$ along the direction of $\boldsymbol{K}_{f b}$, while the transverse components $\boldsymbol{q}_{\perp}$ (perpendicular to $\boldsymbol{K}_{f b}$ ) are set equal to zero in the matrix elements.

For the evaluation of $W_{A}^{(1)}$, the variable $q$ is substituted by $q_{0}=q-s$ in order to simplify the arguments of the $\delta$-functions. Within the peaking approximation, we have $q_{\perp}=0$ which corresponds to the approximation $s_{\perp}=-q_{0 \perp}$ which is made in the $s$ integrand, except for the Coulomb potential $1 / s^{2}$. Introducing spherical coordinates $q_{0}=\left(q_{0}, x \equiv \cos \vartheta_{q_{0}}, \varphi_{q_{0}}\right)$ and choosing the $z$-axis parallel to $K_{f b}, W_{\mathrm{A}}^{(1)}$ reduces with the help of (6.16) and the formula

$\int_{0}^{2 \pi} \mathrm{d} \varphi\left[\pi \delta(C-B \cos \varphi) \pm \frac{\mathrm{i}}{C-B \cos \varphi}\right]=\frac{2 \pi}{\left(B^{2}-C^{2} \mp \mathrm{i} \varepsilon \operatorname{sign} C\right)^{1 / 2}}$

with $\varepsilon=+0$, to the following expression (for s states)

$$
\begin{aligned}
& \begin{aligned}
W_{\mathrm{A}}^{(1)}=\frac{1}{4 \pi^{3} \mathrm{i} \mu_{b}} & \int_{0}^{\infty} q_{0}^{2} \mathrm{~d} q_{0} \int_{-1}^{1} \mathrm{~d} x\left\{\left[\pi \delta\left(\Delta E-\frac{v_{b}^{2}}{2}+q_{0} x v_{b}\right)+\frac{\mathrm{i}}{\Delta E-v_{b}^{2} / 2+q_{0} x v_{b}}\right]\right. \\
& \times S_{b}\left(q_{0}, x\right) A_{b a}^{(+)}\left(K_{i a}, \theta\right) \\
& \left.+\frac{v_{a}}{v_{b}} \frac{1}{\left(B_{\mathrm{A}}^{2}-C_{\mathrm{A}}^{2}+\mathrm{i} \varepsilon \operatorname{sign} C_{\mathrm{A}}\right)^{1 / 2}} S_{a}\left(q_{0}, x\right) A_{b a}^{(+)}\left(K_{f a}, \theta\right)\right\}
\end{aligned} \\
& S_{\lambda}\left(q_{0}, x\right)=Z_{\mathrm{PA}} \int_{-\infty}^{\infty} \mathrm{d} s_{z} M_{1 \lambda}\left(q_{0}, s_{z}, x\right)\left(\int \frac{\mathrm{d} s_{\perp}}{s_{z}^{2}+s_{\perp}^{2}} \varphi_{f}^{*}\left(q_{0}+s-v_{f b}\right)\right) \\
& B_{\mathrm{A}}=q_{0} v_{a} \sin \theta \sqrt{1-x^{2}} \quad C_{\mathrm{A}}=\Delta E-\frac{v_{a}^{2}}{2}+q_{0} x v_{a} \cos \theta \\
& M_{1 \lambda}\left(q_{0}, s_{z}, x\right)=\left\langle\varphi_{\left(q_{0 z}+s_{z}\right) e_{z}}^{(\lambda)}\left(r_{\mathrm{T}}\right)\left|\mathrm{e}^{\mathrm{i}\left(-q_{0 \perp}+s_{z} e_{z}\right) r_{\mathrm{T}}}\right| \varphi_{i}\left(r_{\mathrm{T}}\right)\right\rangle .
\end{aligned}
$$

In the reaction amplitudes, the influence of the electronic momenta on the scattering angle $\theta$ has been neglected, such that $\theta$ equals the angle between $\boldsymbol{K}_{\mathrm{ia}}$ and $\boldsymbol{K}_{f b}, \theta_{\mathbf{K}_{i a}, \boldsymbol{K}_{f b}}$.

For the evaluation of $W_{\mathrm{B}}^{(1)}$, a variable shift is not required. We introduce spherical coordinates $s=\left(s, x \equiv \cos \vartheta_{s}, \varphi_{s}\right)$, make the peaking approximation $q_{\perp}=0$, and in addition eliminate the dependence of the reaction amplitude on $s$ with the help of $\Delta K_{2}^{\prime}=0$ which is required by the $\delta$-function. This substitution should also be reasonable for the coorresponding principal value term which is peaked at $\Delta K_{2}^{\prime}=0$. With the help of (6.16) and (7.1), $W_{\mathrm{B}}^{(1)}$ turns into

$$
\begin{aligned}
& W_{\mathrm{B}}^{(1)}=\frac{Z_{\mathrm{P}_{a}}}{4 \pi^{3} \mathrm{i} \mu_{b}} \int_{-\infty}^{\infty} \mathrm{d} q_{z} A_{b a}^{(+)}\left(K_{a}, \theta\right)\left(\int \mathrm{d} q_{\perp} \varphi_{f}^{*}\left(q-v_{f b}\right)\right) \int_{0}^{\infty} \mathrm{d} s \int_{-1}^{1} \mathrm{~d} x M_{1 a}\left(q_{z} e_{z}, s\right) \\
& \times\left(\frac{1}{\left(B_{\mathrm{B}}^{2}-C_{\mathrm{B}}^{2}-\mathrm{i} \varepsilon \operatorname{sign} C_{\mathrm{B}}\right)^{1 / 2}}+\frac{v_{a}}{v_{b}} \frac{1}{\left(B_{\mathrm{B}}^{2}-C_{\mathrm{B}}^{2}+\mathrm{i} \varepsilon \operatorname{sign} C_{\mathrm{B}}\right)^{1 / 2}}\right) \\
& B_{\mathrm{B}}=s v_{a} \sin \theta \sqrt{1-x^{2}} \quad C_{\mathrm{B}}=\Delta E-\frac{v_{b}^{2}}{2}+q_{z} v_{b}-s v_{a} x \cos \theta .
\end{aligned}
$$


The matrix element $M_{1 a}\left(q_{z} e_{z}, s\right)$ is defined below (6.7) with $\beta$ set equal to zero, and $K_{a}=K_{f a}-q_{z} v_{b} / v_{a}+v_{b}^{2} / 2 v_{a}-v_{a} / 2$.

The sticking contribution $W_{\mathrm{C}}^{(1)}$ is evaluated in the same way as $W_{\mathrm{B}}^{(1)}$. The resulting expression is

$$
\begin{aligned}
& W_{\mathrm{C}}^{(1)}=-\frac{Z_{\mathrm{Pa}}}{4 \pi^{3} \mathrm{i} \mu_{b}} \int_{-\infty}^{\infty} \mathrm{d} q_{z} A_{b a}^{(+)}\left(K_{f a}-v_{a} / 2-v_{b}^{2} / 2 v_{a}, \theta\right)\left(\int \mathrm{d} q_{\perp} \varphi_{f}^{*}\left(q-v_{f b}\right)\right) \\
& \quad \times \int_{0}^{\infty} \mathrm{d} s \int_{-1}^{1} \mathrm{~d} x M_{1 a}\left(q_{z} e_{z}, s\right)\left(\frac{1}{\left(B_{\mathrm{C}}^{2}-C_{\mathrm{C}}^{2}-\mathrm{i} \varepsilon \operatorname{sign} C_{\mathrm{C}}\right)^{1 / 2}}\right. \\
& \left.+\frac{v_{a}}{v_{b}} \frac{1}{\left(B_{\mathrm{C}}^{2}-C_{\mathrm{C}}^{2}+\mathrm{i} \varepsilon \operatorname{sign} C_{\mathrm{C}}\right)^{1 / 2}}-2 \sqrt{\frac{v_{a}}{v_{b}}} \frac{\theta\left(B_{\mathrm{C}}-\mid C_{\mathrm{C}}\right)}{\sqrt{B_{\mathrm{C}}^{2}-C_{\mathrm{C}}^{2}}}\right) \\
& \mathrm{B}_{\mathrm{C}}=\mathrm{B}_{\mathrm{B}} \quad \mathrm{C}_{\mathrm{C}}=\Delta E+v_{b}^{2} / 2-\operatorname{si}_{a} x \cos \theta .
\end{aligned}
$$

Note that without the last term which is proportional to the Heaviside step function $\theta(y), W_{\mathrm{C}}^{(1)}$ would just compensate $W_{\mathrm{B}}^{(1)}$ in the full peaking approximation with $q_{z}=v_{b}$.

We turn now to the remaining terms $W_{f i}^{(0)}, W_{\mathrm{S}}^{(1)}$ and $W_{f i}^{(01)}$ of the capture amplitude. They contain three-dimensional integrals which are readily evaluated without a peaking approximation. With the help of (6.16), the sticking term $W_{\mathrm{S}}^{(1)}$ reduces for spherical electronic states to

$$
\begin{aligned}
& W_{\mathrm{S}}^{(1)}=\frac{\mathrm{i}}{2 \pi \mu_{b}} \int_{0}^{\infty} q^{2} \mathrm{~d} q \int_{-1}^{1} \mathrm{~d} x \varphi_{f}^{*}\left(q-v_{f b}\right)\left\{\left[Z_{\mathrm{Pb}} M_{2 b}(q) A_{b a}^{(+)}\left(K_{i a}, \theta\right)\left(1-2 \sqrt{\frac{v_{a}}{v_{b}}}\right)\right.\right. \\
&\left.+Z_{\mathrm{P} a} M_{2 a}(q) \frac{v_{a}}{v_{b}} A_{b a}^{(+)}\left(K_{a}, \theta\right)\right] \pi \delta\left(\Delta E-v_{b}^{2} / 2+q v_{f b}\right) \\
&+\left[Z_{\mathrm{Pb}} M_{2 b}(q) A_{b a}^{(+)}\left(K_{i a}, \theta\right)-Z_{\mathrm{P} a} M_{2 a}(q) \frac{v_{a}}{v_{b}} A_{b a}^{(+)}\left(K_{a}, \theta\right)\right] \\
&\left.\times \frac{\mathrm{i}}{\Delta E-v_{b}^{2} / 2+q v_{f b}}\right\} \\
& M_{2 \lambda}(q)=\left\langle\varphi_{q}^{(\lambda)}\left(\boldsymbol{r}_{\mathrm{T}}\right)\left|1 / r_{\mathrm{T}}\right| \varphi_{i}\left(\boldsymbol{r}_{\mathrm{T}}\right)\right\rangle .
\end{aligned}
$$

As concerns the recoil term we have found that in contrast to $W_{\mathrm{S}}^{(1)}$, the dominant contribution to the integral arises from a very narrow region around $q_{z}=v_{b}$, like in the case of $W_{\mathrm{A}}^{(1)}, W_{\mathrm{B}}^{(1)}$ and $W_{\mathrm{C}}^{(1)}$, typically (for $1 \mathrm{~s}$ states) $0.2 v_{b} \leqslant q_{z} \leqslant 2 v_{b}$. Larger values of $q_{z}$ are not only suppressed by the occurrence of $\varphi_{f}^{*}\left(q-v_{f b}\right)$, but also by $M_{3 \lambda}(q) \equiv$ $\tilde{M}_{3 \lambda}(q) q /\left(Z_{\text {T } a}^{2}+q^{2}\right)^{3}$ (again for a 1 s state) which is likewise peaked at $q_{\perp}=0$. Hence we have used the transverse peaking approximation for the recoil term, but retained both $\varphi_{f}^{*}$ and $\left(Z_{T a}^{2}+q^{2}\right)^{-3}$ in the $q_{\perp}$ integral. Then, $W_{f i}^{(0)}$ reduces to

$$
\begin{aligned}
& W_{\tilde{f}}^{(0)}=-\frac{\mathrm{i}}{4 \pi^{2} \mu_{b}} \frac{v_{a}^{3 / 2}}{v_{b}^{1 / 2}} \frac{M_{\mathrm{P} a}}{M_{\mathrm{Pa} a}+M_{\mathrm{T} a}} \int_{-\infty}^{\infty} q_{z} \mathrm{~d} q_{z}\left(\int \mathrm{d} q_{\perp} \varphi_{f}^{*}\left(q-v_{f b}\right) \frac{1}{\left(Z_{\mathrm{T} a}^{2}+q^{2}\right)^{3}}\right) \\
& \times\left[\tilde{M}_{3 b}\left(q_{z}\right) A_{b a}^{(+)}\left(K_{i a}, \theta\right)-\tilde{M}_{3 a}\left(q_{z}\right) \cos \theta A_{b a}^{(+)}\left(K_{a}, \theta\right)\right] .
\end{aligned}
$$

The surface term $W_{f i}^{(01)}$ is again evaluated without approximations. With $\Delta K_{6}^{\prime}=$ $-\Delta K_{6} v_{a} / v_{b}=\left(\Delta E+v_{b}^{2} / 2\right) / v_{b}>0$, the $\delta$ function in the sticking contribution vanishes 
and one is left with

$$
\begin{aligned}
W_{f}^{(01)}=-\frac{\mathrm{i} Z_{\mathrm{Pb}}}{4 \pi^{2} \mu_{b}} A_{b a}^{(+)}\left(K_{i a}, \theta\right) \int_{-\infty}^{\infty} \mathrm{d} q_{z}\left[\pi \delta\left(\Delta E-\frac{v_{b}^{2}}{2}+q v_{f b}\right)+\frac{\mathrm{i}}{\Delta E-v_{b}^{2} / 2+q v_{f b}}\right] \\
\times \int \mathrm{d} q_{\perp} \varphi_{i}(q) M_{4}(q)-\frac{1}{4 \pi^{2} \mu_{b}} M_{S} \\
\times\left[Z_{\mathrm{Pb}} A_{b a}^{(+)}\left(K_{i a}, \theta\right)-Z_{\mathrm{Pa}} \frac{v_{a}}{v_{b}} A_{b a}^{(+)}\left(K_{j a}-v_{a} / 2-\frac{v_{b}^{2}}{2 v_{a}}, \theta\right)\right] \frac{1}{\Delta E+v_{b}^{2} / 2}
\end{aligned}
$$

With the explicit formulae for the various contributions to the transition amplitude $W_{f i}^{b a}$, equations $(6.1)$ or $(6.17)$, the capture probability at a given scattering angle $\theta$ is readily calculated from

$$
\begin{aligned}
& P_{b a}(\theta)=(2 \pi)^{4} \mu_{b}^{2} \frac{v_{b}}{v_{a}} N_{0} \\
& \times \frac{\sum_{m_{s_{\mathrm{p}}} m_{s_{\mathrm{T}}}} \sum_{m_{s_{\mathrm{p}}}^{\prime} m_{s_{\mathrm{T}}}^{\prime}}\left|W_{f i}^{b a}\left\{A_{b a}^{(+)}\left(m_{s_{\mathrm{P}}}, m_{s_{\mathrm{T}}}, m_{s_{\mathrm{P}}}^{\prime}, m_{s_{\mathrm{T}}}^{\prime} ; E_{a}, \theta\right)\right\}\right|^{2}}{\sum_{m_{s_{\mathrm{P}}} m_{s_{\mathrm{T}}}} \sum_{m_{s_{\mathrm{p}}}^{\prime} m_{s_{\mathrm{T}}}^{\prime}}\left|A_{b a}^{(+)}\left(m_{s_{\mathrm{p}}}, m_{s_{\mathrm{T}}}, m_{s_{\mathrm{p}}}^{\prime}, m_{s_{\mathrm{T}}}^{\prime} ; E_{i a}, \theta\right)\right|^{2}}
\end{aligned}
$$

where it is summed over the polarization directions of the two nuclei, and the dependence of the reaction amplitude (3.3) on the corresponding magnetic quantum numbers is explicitly indicated. $N_{0}$ is the number of electrons in the initial target state, and $W_{f i}^{b a}\{\ldots\}$ denotes the right-hand side of (6.17). The denominator in (7.8) is the nuclear reaction cross section (multiplied by $\left(2 s_{\mathrm{P}}+1\right)\left(2 s_{\mathrm{T}}+1\right)$ ). Hence, ${ }_{p b \alpha}(\theta)$ is the probability for detecting ejectiles with a captured electron relative to the number of bare ejectiles produced in the reaction. Alternatively, one may define a capture probability, say $P_{b a}^{(a)}(\theta)$, where the denominator in (7.8) is replaced by the nuclear elastic scattering cross section, defined by squaring the scattering amplitude $A_{a a}^{(+)}\left(E_{i a}, \theta\right)$ instead of $A_{b a}^{(+)}\left(E_{i a}, \theta\right)$. With this definition, $P_{b a}^{(a)}$ is the probability for finding one-electron ejectiles relative to the number of elastically scattered projectiles, and hence includes the probability that the nuclear reaction takes place.

So far, the formalism has been developed for nuclear reactions. The results are, however, easily modified to include also the case of elastic scattering. The capture probability in the elastic channel $a$ is given by

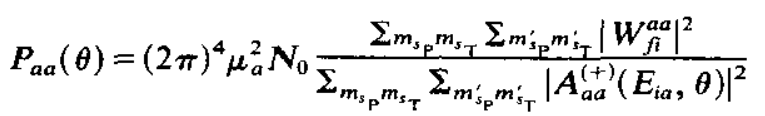

where $A_{a a}^{(+)}\left(E_{a}, \theta\right)$ is obtained from (3.3) with $s_{\mathrm{P}}=s_{\mathrm{P}}^{\prime}, s_{\mathrm{T}}=s_{\mathrm{T}}^{\prime}, l=l^{\prime}$ and the formal replacement of indices $b$ by $a$, upon adding the contribution from the Coulomb scattering amplitude, $f_{\text {Coul }}\left(K_{a}, \theta\right) \delta m_{s_{\mathrm{p}}} m_{s_{\mathrm{p}}}^{\prime} \delta m_{s_{\mathrm{r}}}, m_{s_{\mathrm{T}}^{\prime}}$ (Lane and Thomas 1958). The channel$a$ capture amplitude $W_{f i}^{a a}$ is found from (6.1) or (6.17) by replacing everywhere the index $b$ by $a$ and setting the $Q$-value equal to zero. With this procedure, the one-channel formulae of JAA are recovered to a large extent. There are basically four differences between the previous and the present formulation of the theory. (i) Instead of SPB, the impulse approximation is used; this should, however, be of minor importance at the higher collision energies (Jakubassa-Amundsen 1984, Taulbjerg et al 1990). (ii) Instead of outgoing intermediate nuclear waves, incoming waves $\psi_{a K}^{(+)}$are used in channel $a$ (see equation (4.2)). This avoids the occurrence of spurious complex conjugate scattering amplitudes, but introduces an additional $S$-matrix contribution to the sticking term $W_{C}^{(1)}$ (the term $W_{a}(0)$ in $(6.12)$ ). (iii) In JAA, the surface term-which tends to cancel 
$W_{a}(0)$ to some extent-has been omitted. (iv) Instead of the full peaking, the transverse peaking approximation has been applied. This should be responsible for the main part of the deviations between the two theories.

In order to test the formalism and the peaking approximation we have reinvestigated the capture probability for protons colliding elastically with carbon and have compared with previous calculations and with the experimental data from Scheurer et al (1985) and Horsdal Pedersen (1987). Figure 1 displays the angular dependence of the capture probability at collision energies of $0.5 \mathrm{MeV}$ and $1 \mathrm{MeV}$. Shown are previous results in the fully peaked SPB theory without the surface term, and with the surface term inciuded. Both these theories are seriously at variance with the data, especially at backward angles. Our present results give a considerable improvement, although at the lower energy the data are still overestimated. It should be noted in this context that the peaking approximation gets more accurate, the higher the collision energy.

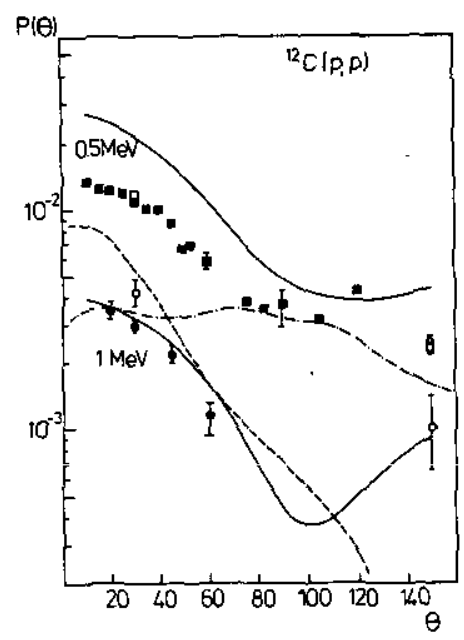

Figure 1. Capture probability for ${ }^{12} \mathrm{C} \mathrm{K}$-shell electrons by $0.5 \mathrm{MeV}$ and $1 \mathrm{MeV}$ protons as a function of scattering angle. Full curves denote the transverse-peaked IA results, the chain curve is the fully peaked SPB result from JAA and the broken curve is the SPB result with the surface term included (from Jakubassa-Amundsen 1987), both for $E_{\mathrm{p}}=1 \mathrm{MeV}$. The experimental data are from Scheurer et al (1985) and from Meyerhof (private communication) $\left(\diamond, E_{\mathrm{p}}=0.49 \mathrm{MeV} ; \square, E_{\mathrm{p}}=0.51 \mathrm{MeV} ; O, E_{\mathrm{p}}=1 \mathrm{MeV}\right)$ as well as from Horsdal Pedersen (1987) (a, $E_{p}=0.5 \mathrm{MeV} ; 0, E_{p}=1 \mathrm{MeV}$ ).

In figure 2 the energy dependence of the capture probability is shown, including the very broad isolated $s_{1 / 2}$ resonance $\left(E_{R, 1 a b}=0.462 \mathrm{MeV}, \Gamma_{\mathrm{lab}}=35 \mathrm{keV}\right)$. Although the data are again overestimated by the transverse peaked IA, especially at the lower energies, the slope of the data is better reproduced than with the previous fully peaked calculations.

In order to estimate the accuracy of the transverse peaking approximation for nuclear reactions, we have made test calculations for the recoil term $W_{n}^{(0)}$ in the full and the transverse peaking approximation and have found deviations of about $30 \%$. It also turned out that the sum

$$
\left|W_{\mathrm{B}}^{(1)}+\left(W_{\mathrm{C}}^{(1)}-\int \mathrm{d} \boldsymbol{K} \mathrm{d} q W_{\mathrm{a}}(0)\left\langle\psi_{a K}^{(+) \infty}\left|M^{(1)}(\boldsymbol{R})\right| \psi_{a K_{i}}^{(+) \infty}\right\rangle\right)\right|
$$




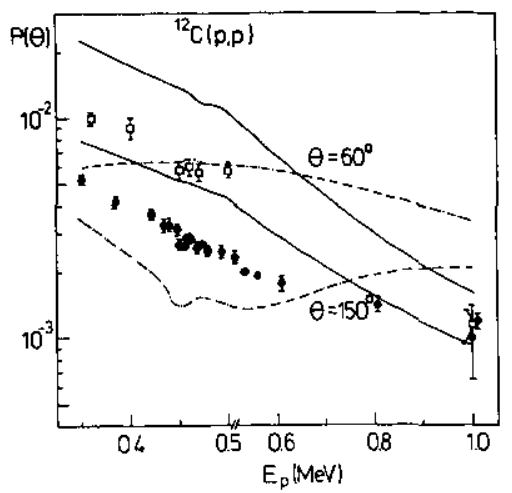

Figure 2. Capture probability for ${ }^{12} \mathrm{C} \mathrm{K}$-shell electrons by protons as a function of proton energy at the scattering angles $\theta=60^{\circ}$ and $150^{\circ}$. Full curves denote transverse-peaked IA results, and the chain curves are the fully peaked SPB calculations from JAA. The experimental data are from Horsdal Pedersen $(1987$; $)$ at $\theta=60^{\circ}$ and from Scheurer et al (1985; \$) at $\theta=150^{\circ}$. For $E_{\mathrm{p}}>0.5 \mathrm{MeV}$, the abscissa is contracted by a factor of 2 .

which vanishes with full peaking, reduces to about $30 \%$ of the individual values in case of transverse peaking. From this we conjecture the accuracy of the transverse peaked IA with respect to the exact IA to be about $10 \%$ for each individual contribution to the transition amplitude (6.1). However, taking into consideration that these contributions add up with different phases, the net accuracy of the transition probability will be worse, maybe even approaching a factor of 2 .

\section{Numerical details of the calculations}

When the spectator electrons of the target are accounted for by means of Slater-screened hydrogenic wavefunctions and experimental binding energies, all matrix elements $M_{1 \lambda}$, $M_{2 \lambda}, M_{3 \lambda}, M_{4}$ and $M_{5}$ can be evaluated analytically and reduce to simple expressions for the case of K-K capture (JAA, Amundsen and Jakubassa-Amundsen 1984b). Also the integrals over the transverse momenta $s_{\perp}$ and $q_{\perp}$ in equations (7.2), (7.3), (7.5), (7.6) and (7.7) reduce to analytical expressions. Hence, the contributions $W_{\mathrm{A}}^{(1)}, W_{\mathrm{B}}^{(1)}$ and $W_{\mathrm{C}}^{(1)}$ involve three-dimensional integrals, while $W_{\mathrm{S}}^{(1)}$ and $W_{f i}^{(0)}, W_{f i}^{(01)}$ contain a two- and one-dimensional integral, respectively, which all have to be evaluated numerically.

The techniques for calculating the various momentum integrals are basically the same. Where principal value terms are involved, an analytical treatment of the pole is required

$$
\begin{aligned}
\int_{0}^{\infty} \mathrm{d} q \int_{-1}^{1} \mathrm{~d} x & \frac{\mathrm{i}}{p+q x} F(q, x) \\
= & \int_{0}^{|p|-\delta} \mathrm{d} q \int_{-1}^{1} \mathrm{~d} x \frac{\mathrm{i}}{p+q x} F(q, x)+\int_{|p|+\delta}^{\infty} \mathrm{d} q \\
& \times\left(\int_{-1}^{1} \mathrm{~d} x \frac{\mathrm{i}}{p+q x}[F(q, x)-F(q,-p / q)]+F(q,-p / q) \frac{\mathrm{i}}{q} \ln \left|\frac{p+q}{p-q}\right|\right)
\end{aligned}
$$


where $F(q, x)$ denotes the slowly varying part of the integrand near the pole at $x=-p / q$, and $\delta$ is a small positive quantity.

If the integrand contains a square root instead of the principal value term, as e.g. in the second contribution to $W_{A}^{(1)}$, an analytical treatment of the singularities is also required. In the case of $W_{\mathrm{A}}^{(1)}$, the two square-root singularities are located at $x=$ $-p \cos \theta / q_{0} \mp \sin \theta\left[1-\left(p / q_{0}\right)^{2}\right]^{1 / 2}$ with $p=\left(\Delta E-v_{a}^{2} / 2\right) / v_{a}$. Only for $q_{0}<|p|$, the poles lie outside the integration region, while for $q_{0}>|p|$, the $x$ integral has to be divided into two parts, each of them containing one pole, and the poles have to be treated in a similar way as indicated by $(8.1)$. The analytical contribution from the poles involves the following integral

$\int \mathrm{d} x \frac{1}{\left(-a x^{2}+b x+c\right)^{1 / 2}}=\frac{\mathrm{i}}{\sqrt{a}} \ln \left[-2 \mathrm{i} \sqrt{a}\left(-a x^{2}+b x+c\right)^{1 / 2}-2 a x+b\right]$

with $a>0$ and $c$ a complex number. With this treatment, the pathological case when the two poles coincide is unproblematic. In the innermost region of the momentum integral $(q<|p|$ in the notation of equation (8.1)), a logarithmic variable substitution, $y=\ln (|p|-q)$ should be made. The numerical accuracy of the calculations is estimated to be about $5 \%$.

\section{Results}

We have evaluated the transition probabilities for electron capture during the nuclear reactions ${ }^{19} \mathrm{~F}(\mathrm{p}, \alpha){ }^{16} \mathrm{O},{ }^{32} \mathrm{~S}\left(\mathrm{p}, \mathrm{p}^{\prime}\right){ }^{32} \mathrm{~S},{ }^{12} \mathrm{C}(\mathrm{d}, \mathrm{p}){ }^{13} \mathrm{C}$ and ${ }^{16} \mathrm{O}(\mathrm{n}, \alpha){ }^{13} \mathrm{C}$, restricting ourselves to $1 \mathrm{~s} \rightarrow 1 \mathrm{~s}$ electronic transitions. With the choice of the above collision systems, we have selected the simplest case of isolated nuclear resonances.

As the first example, we have investigated the nucleon transfer reaction ${ }^{19} \mathrm{~F}\left(\mathrm{p}, \alpha_{i}\right){ }^{16} \mathrm{O}$ with $i=2-4$ which is the only case where electron capture data are available (Horsdal Pedersen 1987). The proton energy required to populate the $J=2$ state of the compound nucleus is $E_{\mathrm{p}}=0.873 \mathrm{MeV}$. This state can decay into a series of three $\alpha$ particles with $Q$-values of $Q_{2}=1.9836 \mathrm{MeV}, Q_{3}=1.194 \mathrm{MeV}$ and $Q_{4}=0.994 \mathrm{MeV}$ and partial laboratory widths $\Gamma_{b 2}=2.2 \mathrm{keV}, \Gamma_{b 3}=0.62 \mathrm{keV}$ and $\Gamma_{b 4}=0.18 \mathrm{keV}$. The total decay width is $\Gamma_{\mathrm{lab}}=4.53 \mathrm{keV}$ (Ajzenberg-Selove 1987, p 163). When correlations between the three different emitted $\alpha$ particles are neglected, the formalism developed above can be applied to each $\alpha_{i}$ separately. We shall restrict ourselves to the calculation of the capture probability in the inelastic channel, equation (7.8). As both numerator and denominator of (7.8) are proportional to the reaction amplitude, the angular momentum dependence of $\boldsymbol{A}_{b a}^{(+)}$drops out because the electronic transition amplitudes are independent of the angular momentum variables. This means that $A_{b a}^{(+)}$in (7.8) can formally be replaced by the simple Breit-Wigner term

$$
A_{b a}^{(+)}\left(E_{a}, \theta\right) \rightarrow \frac{1}{E_{a}-E_{\mathrm{R}}^{(a)}+\mathrm{i} \Gamma / 2}
$$

and the sums over the magnetic quantum numbers in (7.8) can be disregarded. For the ${ }^{19} \mathrm{~F}\left(\mathrm{p}, \alpha_{2}\right)$ reaction, the electronic energy transfer of $1.1 \mathrm{keV}$ is considerably smaller than the total decay width, $\Gamma=4.3 \mathrm{keV}$, of the resonance. Nevertheless, the interference effects manifest themselves in a peak in the impact energy dependence of $P(\theta)$ near the resonance energy, which rises about a factor of 2 above the background for forward scattering angles $\left(\theta \leqslant 60^{\circ}\right)$ and less for larger angles. The peak shape depends only weakly on $\theta$ or on the $Q$-value and is similar as for the reaction in the neighbouring 
system, ${ }^{18} \mathrm{O}(\mathrm{p}, \alpha){ }^{15} \mathrm{~N}$ which was studied in a first investigation (Jakubassa-Amundsen 1990).

Figure 3 shows the capture probability in the ${ }^{19} \mathrm{~F}\left(\mathrm{p}, \alpha_{2}\right)$ reaction at the resonance energy $E_{\mathrm{p}}=0.873 \mathrm{MeV}$ as a function of the scattering angle. $P(\theta)$ drops smoothly by a factor of 3 when $\theta$ is increased from $10^{\circ}$ to $90^{\circ}$, and flattens out for larger angles. Horsdal Pedersen (1987) has suggested that such an angular variation of the capture probability is caused by the interference of the atomic transition amplitudes from the incoming and outgoing part of the collision. In fact, these amplitudes are of comparable magnitude for the ${ }^{19} \mathrm{~F}(\mathrm{p}, \alpha)$ reaction: Although the perturbation $V_{\mathrm{P}}$ is much weaker for the proton channel than for the $\alpha$ channel, this is compensated by the subsequent overlap with the $\mathrm{He}^{+}$final state. In order to compare with 'full-trajectory' transition probabilities, the capture probability for the elastic ${ }^{16} \mathrm{O}(\alpha, \alpha)$ collision (without consideration of a resonance) is also shown. We have chosen a projectile energy, $E_{\alpha}=$ $3.5 \mathrm{MeV}$, which is equal to the impact energy of the inverse reaction at the resonance position, ${ }^{16} \mathrm{O}(\alpha, \mathrm{p}){ }^{19} \mathrm{~F}$, with the ${ }^{16} \mathrm{O}$ nucleus at rest. This energy is calculated from the formula (Baldin et al 1963)

$$
E_{\alpha}=\frac{M_{\mathrm{P} b}+M_{\mathrm{T} b}}{M_{\mathrm{T} b}}\left(\frac{M_{\mathrm{T} a}}{M_{\mathrm{P} a}+M_{\mathrm{T} a}} E_{\mathrm{p}}+Q\right) .
$$

The capture probabilities for ${ }^{16} \mathrm{O}(\alpha, \alpha)$ and ${ }^{19} \mathrm{~F}\left(\mathrm{p}, \alpha_{2}\right)$ are of similar magnitude because $v_{b}$ and hence the impact velocity for $\alpha+{ }^{16} \mathrm{O}$ is very close to $v_{a}$. However, the constructive interference of the atomic amplitudes in the non-resonant $(\alpha, \alpha)$ collision leads to a stronger angular variation of $P(\theta)$ than for the $\left(\mathrm{p}, \alpha_{2}\right)$ reaction.

While theory overestimates experiment in case of the elastic ${ }^{16} \mathrm{O}(\alpha, \alpha)$ collision (like for the $\mathbf{p}+\mathrm{C}$ system, figure 2), it falls considerably below the data for the nuclear reaction. Following the suggestion of Horsdal Pedersen (1987) we have tentatively assumed that after the reaction, the electron cloud sticks to the centre-of-mass rather than to the recoiling ${ }^{16} \mathrm{O}$ nucleus. This would imply a reduction of $v_{b}$ from 5.9 au to $4.8 \mathrm{au}$, with a corresponding increase of the capture probability of about $50 \%$. This is, however, still too small to account fully for the deviations from experiment.

The dependence of $P(\theta)$ on the $Q$-value, i.e. on the energy of the emitted $\alpha$ particle, is depicted in figure 4 and compared with the energy dependence of the non-resonant

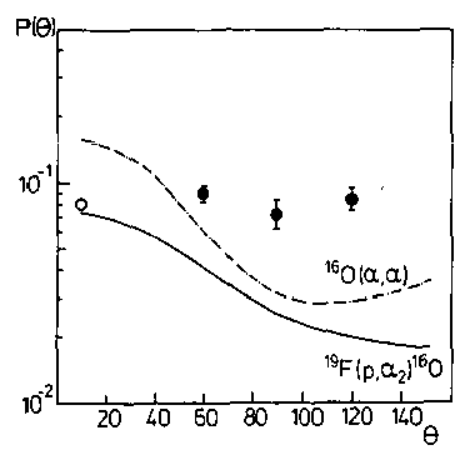

Figure 3. Electron capture probability during the reaction ${ }^{19} \mathrm{~F}\left(\mathrm{p}, \alpha_{2}\right){ }^{16} \mathrm{O}$ at the resonance energy $E_{\mathrm{p}}=0.873 \mathrm{MeV}$ as a function of scattering angle. The full curve is an IA calculation for capture from the ${ }^{19} \mathrm{~F} \mathrm{~K}$ shell, and the experimental data $(\mathcal{)})$ are from Horsdal Pedersen (1987). The chain curve denotes IA results for K-shell capture from ${ }^{16} \mathrm{O}$ by $\alpha$ impact at $E_{a}=3.5 \mathrm{MeV}$. The data point for this system (O) is from Horsdal Pedersen (1987). 


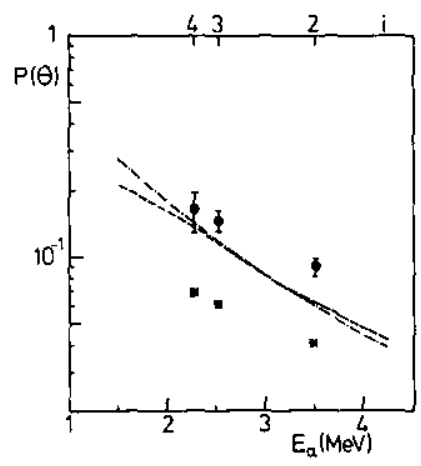

Figure 4. Capture probability during the reaction ${ }^{19} \mathrm{~F}\left(\mathrm{p}, \alpha_{i}\right)^{16} \mathrm{O}$ for $\theta=60^{\circ}$ at the resonance energy $E_{\mathrm{p}}=0.873 \mathrm{MeV}$ as a function of the $Q$-value (upper scale). Theoretical K-shell iA results are denoted by an asterisk $(*)$, and the experimental data $(\boldsymbol{\phi})$ are from Horsdal Pedersen (1987). Also shown are the theoretical results for $\alpha$ impact on the residual nucleus ${ }^{16} \mathrm{O}$ (chain curve) and on the target ${ }^{19} \mathrm{~F}$ (broken curve) as a function of $\alpha$-energy (lower scale).

${ }^{16} \mathrm{O}(\alpha, \alpha)$ and ${ }^{19} \mathrm{~F}(\alpha, \alpha)$ collisions. The capture probability for the reaction lies $50-100 \%$ below the results for the elastic collisions and has a similar $Q$-value dependence as the experimental data. The decrease of $P(\theta)$ with increasing $E_{\alpha}$ is related to the growing energy transfer to the electron.

The reaction ${ }^{12} \mathrm{C}\left(\mathrm{d}, \mathrm{p}_{0}\right){ }^{13} \mathrm{C}$ at the resonance energy $E_{\mathrm{d}}=1.4495 \mathrm{MeV}$ differs from the previous reaction in two points: The perturbation $V_{\mathrm{P}}$ is the same in both channels, but the collision velocities are considerably different, $v_{b}=2.4 v_{a}$. The decay width of the $J=2$ compound nuclear state is $\Gamma=7 \mathrm{keV}$ (Tryti et al 1973). The partial decay widths for the inelastic $\left(d, p_{0}\right)$ channel $\left(\Gamma_{b}=2.1 \mathrm{keV}\right)$ and for the elastic $(d, d)$ channel $\left(\Gamma_{a}=3.8 \mathrm{keV}\right)$ are derived from the corresponding excitation functions (Jeronymo $e t$ al 1963). The $Q$-value of the reaction is $2.7218 \mathrm{MeV}$. For this system, the three probabilities $P_{a a}(\theta)$, equation (7.9), $P_{b a}(\theta)$, equation (7.8) as well as the reaction probability $P_{b a}^{(a)}(\theta)$, which is normalized to the elastic scattering cross section, are investigated. Correspondingly, the reaction amplitude has to be calculated from (3.3). For channel $a$, the angular momentum variables are $s_{\mathrm{P}}=1, s_{\mathrm{T}}=0, s=1$ and $l=2$, while for channel $b, s_{\mathrm{P}}^{\prime}=\frac{1}{2}, s_{\mathrm{T}}^{\prime}=\frac{1}{2}, s^{\prime}=1$ and $l^{\prime}=1$. In the expression for $P_{a a}(\theta)$, six terms contribute to the sums in (7.9), which can, however, be combined to three terms, one, where $A_{a a}^{(+)}$contains only the Breit-Wigner term, the second, where $A_{a a}^{(+)}$contains only the Coulomb scattering amplitude, and the third, where $A_{a a}^{(+)}$contains both. Consequently, $W_{f i}^{a a}$ has to be calculated separately for these three cases. On the other hand, the four terms which contribute to the numerator of $P_{b a}(\theta)$ in (7.8) can be combined to a single term because in $A_{b a}^{(+)}$, the Coulomb scattering amplitude is always absent.

Figure 5 shows the energy dependence of the capture probabilities at a scattering angle of $60^{\circ}$. For the elastic channel, the energy transfer $\Delta E+v_{a}^{2} / 2=0.665 \mathrm{keV}$ is only one tenth of $\Gamma$ and hence, no resonance structure is observable. For the neutron transfer reaction, the corresponding energy transfer is $2.6 \mathrm{keV}$ and the excursion of $P_{b a}$ across the resonance is a factor of 4 . However, due to the large energy transfer in the inelastic channel because of the large value of $v_{b}, P_{b a}(\theta)$ is more than one order of magnitude below $P_{a a}(\theta)$. The reaction probability $P_{b a}^{(a)}(\theta)$ is peaked near the resonance energy with a steep fall-off at the wings, and displays basically the Breit-Wigner-shaped 


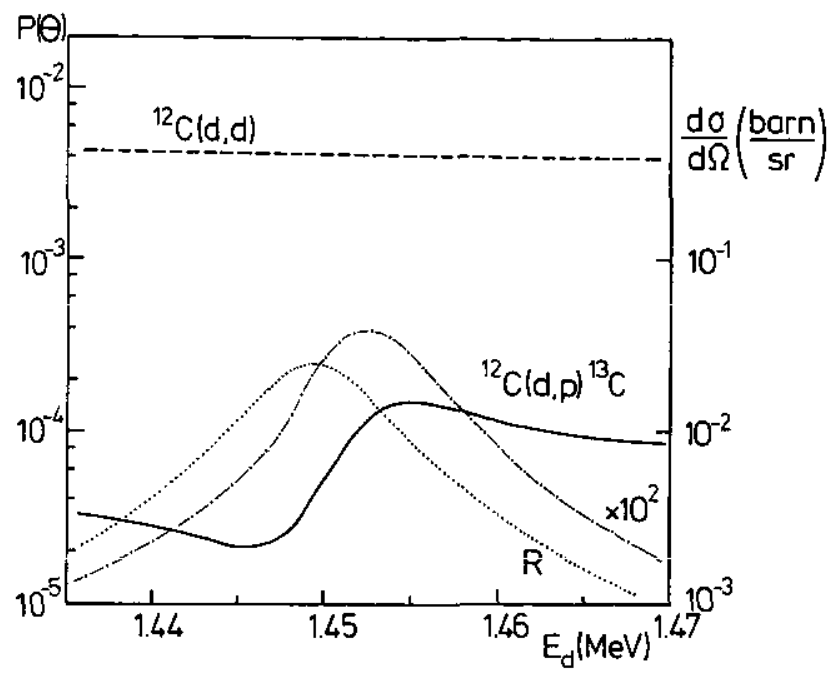

Figure 5. Capture probability (left-hand scale) for carbon K-shell electrons as a function of deuteron energy in the reaction ${ }^{12} \mathrm{C}(\mathrm{d}, \mathrm{p}){ }^{13} \mathrm{C}$ when normalized to the nuclear reaction cross section ( - ) and when normalized to the elastic scattering cross section (- $--\cdot-$; multiplied by $\left.10^{2}\right)$. Also shown is the capture probability for the elastic channel ${ }^{\mathrm{i} 2} \mathrm{C}(\mathrm{d}, \mathrm{d})$ $(-\ldots)$. The right-hand scale is for the reaction cross section (dotted curve $R$ ). The scattering angle is $60^{\circ}$.

probability for the $\left(\mathrm{d}, \mathrm{p}_{0}\right)$ reaction to occur. For comparison, the nuclear reaction cross section is also shown in figure 5. As concerns the angular dependence of $P_{b a}^{(a)}(\theta)$, the peak intensity drops strongly for $\theta<60^{\circ}$ and increases weakly for $\theta>90^{\circ}$, according to the behaviour of the Coulomb scattering amplitude contained in $\boldsymbol{A}_{a a}^{(+)}$. Consequently, the absolute intensity of ejectiles with a captured electron decreases strongly for scattering angles below $60^{\circ}$ and for impact energies $E_{a}$ with $\left|E_{a}-E_{\mathrm{R}}^{(a)}\right|>\Gamma$.

As example of an inelastic scattering without nucleon transfer we have studied the collision system ${ }^{32} \mathrm{~S}\left(\mathrm{p}, \mathrm{p}^{\prime}\right)$ in the vicinity of the resonance at $E_{\mathrm{p}}=3.716 \mathrm{MeV}$. The $J=\frac{5}{2}$ compound state decays with a width of $\Gamma_{\mathrm{jab}}=1.5 \mathrm{keV}$ into the elastic channel $\left(\Gamma_{a, \mathrm{iab}}=\right.$ $0.84 \mathrm{keV})$ and one inelastic channel $\left(\Gamma_{b, \mathrm{lab}}=0.66 \mathrm{keV}\right)$ where an excited state of the ${ }^{32} \mathrm{~S}$ nucleus is populated. Consequently, the $Q$-value is negative, $Q=-2.237 \mathrm{MeV}$ (Endt and van der Leun 1973, p 310). The channel- $a$ angular momentum variables are $s_{\mathrm{P}}=\frac{1}{2}$, $s_{\mathrm{T}}=0, s=\frac{1}{2}$ and $l=3$, whereas for channel $b, s_{\mathrm{P}}^{\prime}=\frac{1}{2}, s_{\mathrm{T}}^{\prime}=2, l^{\prime}=1$ and hence there are two allowed channel spins, $s^{\prime}=\frac{3}{2}$ and $\frac{5}{2}$. The experimentally determined occupation probability for $s^{\prime}=\frac{5}{2}$ is 0.9 , and 0.1 for $s^{\prime}=\frac{3}{2}$ (Olness et al 1958), and the corresponding capture probabilities must be multiplied by these weight factors before being added. For capture in the elastic channel, the terms contributing to the sums in (7.9) can effectively be combined to two terms, one where $\boldsymbol{A}_{a a}^{(+)}$contains only the Breit-Wigner part (the spin-flip term), and the other where the Coulomb amplitude is additionally present in $A_{a a}^{(+)}$(the 'non-flip' term).

In contrast to the reactions studied previously, the energy transfer in the elastic channel $(4.48 \mathrm{keV})$ and the inelastic channel $(3.23 \mathrm{keV})$ exceeds the width $\Gamma$ considerably. This leads to strong variations of the reaction amplitude as a function of $E_{a}$ in the vicinity of $E_{\mathrm{R}}^{(a)}$, which are much more prominent than in the elastic scattering amplitude where they are damped by the slowly varying Coulomb contribution. In fact, as depicted in figure $6, P_{b a}(\theta)$ changes by nearly two orders of magnitude when 


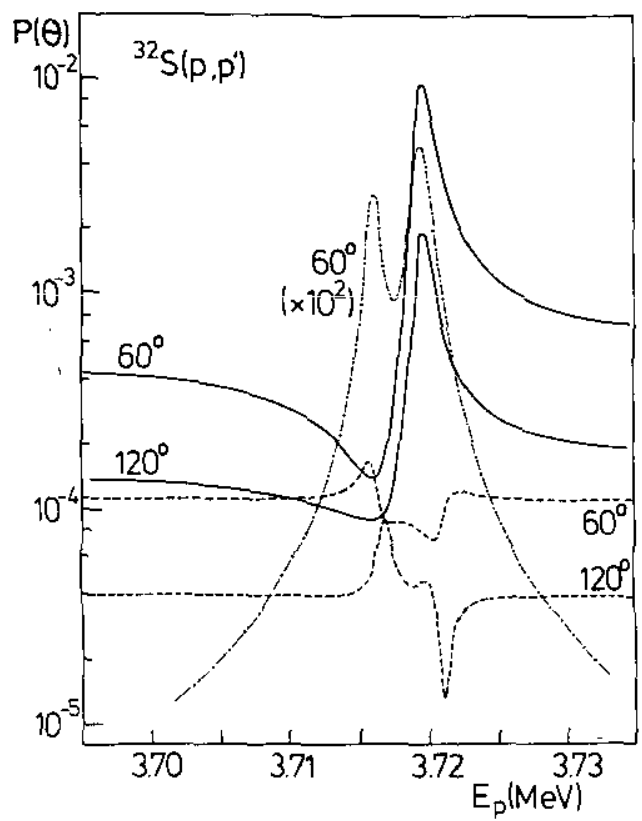

Figure 6. Capture probability (left-hand scale) for ${ }^{32} \mathrm{~S} \mathrm{~K}$-shell electrons by protons as a function of proton energy at the scattering angles $\theta=60^{\circ}$ and $120^{\circ}$. Shown are results for the elastic channel (-- - ), as well as for the inelastic channel normalized to the reaction cross section (-) and to the elastic scattering cross section (.-- - ; multiplied by $10^{2}$ ), respectively. Also shown is the reaction cross section (dotted curve $R$, right-hand scale).

$E_{\mathrm{p}}$ is varied across the resonance, both for forward and backward scattering angles. The excursion of $P_{a a}(\theta)$ is much weaker, at most a factor of 3 . However, there is a 'doubling' of the structure which arises primarily from the interference between the Coulomb part and the Breit-Wigner part in the 'non-flip' term of $A_{a a}^{(+)}$. The energy position of this interference structure is different for the partial transition amplitude where electron transfer has taken place before nuclear scattering (i.e. where the impact energy is reduced by the electronic energy transfer) and where the scattering occurs first. Consequently, the separation of the two peaks in $P_{a a}$ or $P_{b a}^{(a)}$ is given by the energy transfer. As the width of the peaks is determined by $\Gamma$, a necessary condition for the visibility of the structure doubling is $\Delta E+v_{a}^{2} / 2 \gg \Gamma$. This is similar to the case of the historical molecular resonance in ${ }^{8} \mathrm{Be}$ (Benn et al 1968, Heinz 1987). In contrast to $P_{b a}^{(a)}$, the nuclear reaction cross section (also shown in figure 6) has only a single maximum.

The dependence of $P_{b a}(\theta)$ on the scattering angle is again monotonic (figure 7). In the elastic channel, the presence of the Coulomb amplitude causes strong angular variations of $P_{a a}(\theta)$, and the two-dip structure refiects the corresponding structure in the energy dependence. The reaction probability $P_{b a}^{(a)}$ shows the increase with $\theta$ (for $\theta<120^{\circ}$ ) which is related to the angular variation of the Coulomb scattering amplitude, but then it decreases again strongly. We attribute this decrease also to the 'nuclear' interference effects.

In the last example, the case of a typical half-trajectory capture probability is studied by investigating the neutron-induced reaction ${ }^{16} \mathrm{O}(\mathrm{n}, \alpha){ }^{13} \mathrm{C}$ near the resonance at $E_{n}=4.6318 \mathrm{MeV}$. The $J=\frac{3}{2}$ compound state has a total width of $\Gamma=6.89 \mathrm{keV}$, and 


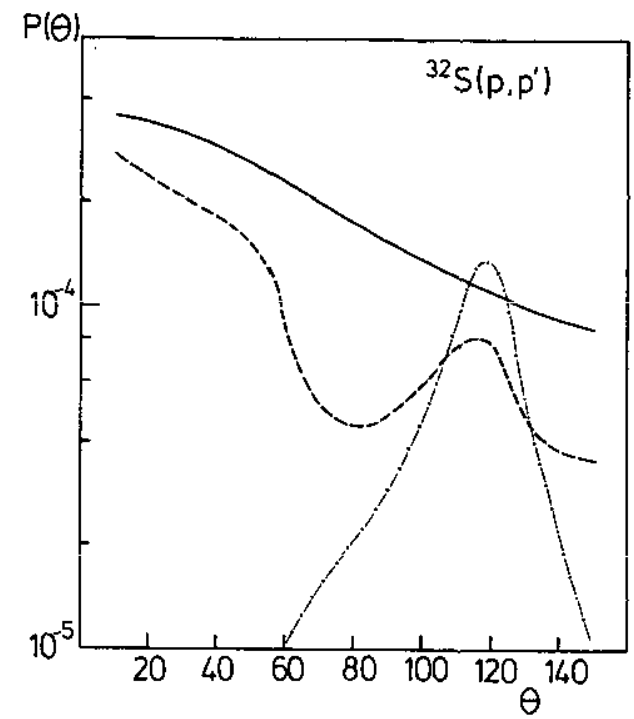

Figure 7. Capture probability for ${ }^{32} \mathrm{~S} \mathrm{~K}$-shell electrons by protons as a function of scattering angle at the near-resonance energy $E_{\mathrm{p}}=3.717 \mathrm{MeV}$. The meaning of the curves is the same as in figure 6.

a partial decay width $\Gamma_{b}=1.9 \mathrm{keV}$, while the $Q$-value of the reaction is $-2.2156 \mathrm{MeV}$ (Ajzenberg-Selove 1986, p 89). Since capture only takes place in the inelastic channel, the reaction amplitude can again be represented by the simple expression (9.1). The fact that $Z_{\mathrm{P}}=0$ in channel $a$ makes all contributions to the transition amplitude vanish except those where electron transfer (ionization + capture) takes place after the nuclear reaction, or where the transition is induced by recoil. It is just this recoil contribution which is present both in the incoming and outgoing part of the collision and which

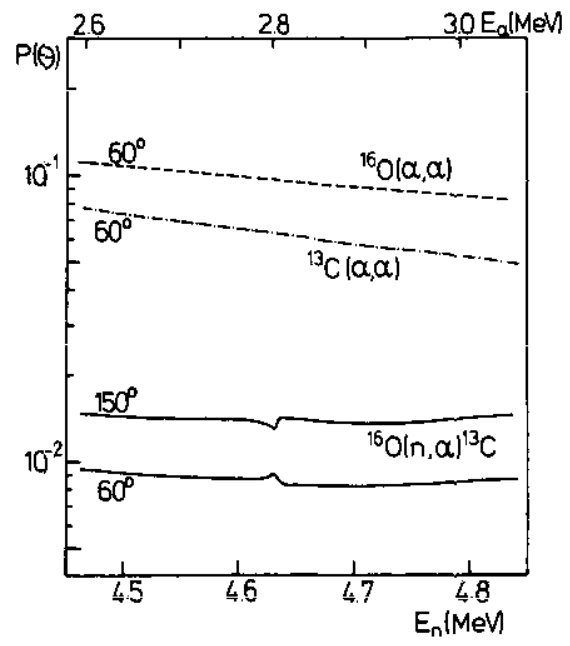

Figure 8. K-shell capture probability by $\alpha$ particles from the reaction ${ }^{16} \mathrm{O}(\mathrm{n}, \alpha){ }^{13} \mathrm{C}$ as a function of neutron energy (lower scale) at $\theta=60^{\circ}$ and $150^{\circ}$. Also shown are results for $\alpha$ impact on the residual nucleus ${ }^{13} \mathrm{C}(-.-.-)$ and on the target ${ }^{16} \mathrm{O}(---)^{-}$at $\theta=60^{\circ}$ as a function of $\alpha$-energy (upper scale). 


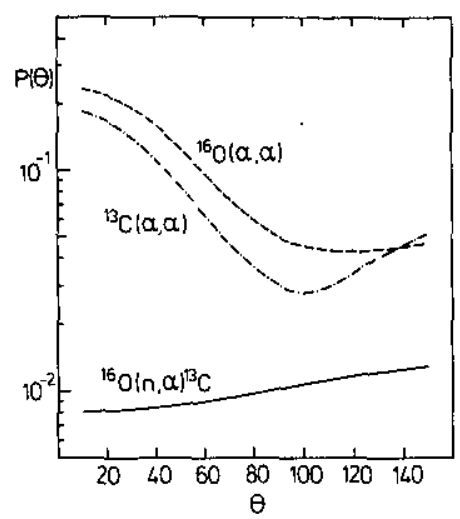

Figure 9. K-shell capture probability by $\alpha$ particles as a function of scattering angle. The full curve denotes the capture probability for the reaction ${ }^{16} \mathrm{O}(n, \alpha){ }^{13} \mathrm{C}$ at the resonance energy $E_{\mathrm{n}}=4.63 \mathrm{MeV}$, while the broken and chain curves give results for $\alpha$ particles colliding with ${ }^{16} \mathrm{O}$ and ${ }^{13} \mathrm{C}$, respectively, at an impact energy $E_{\alpha}=2.8 \mathrm{MeV}$.

can produce interference effects. This 'recoil-interference' which leads to a peak (at $\theta=60^{\circ}$ ) or dip (at $150^{\circ}$ ) in the energy dependence of $P_{b a}(\theta)$ is, however, rather weak (figure 8). In this figure, comparison is also made with the non-resonant capture amplitudes from the collisions ${ }^{13} \mathrm{C}(\alpha, \alpha)$ and ${ }^{16} \mathrm{O}(\alpha, \alpha)$ where the $\alpha$-impact energy $E_{\alpha}$ is related to $E_{\mathrm{n}}$ by $(9.2)$ with $E_{\mathrm{p}}$ replaced by $E_{\mathrm{n}}$. The capture probabilities for the $\alpha+{ }^{16} \mathrm{O}$ and $\alpha+{ }^{13} \mathrm{C}$ systems lie about one order of magnitude above the neutron-induced capture probability and hence should be interpreted cautiously as the corresponding 'full-trajectory' capture probabilities: while in the $\alpha+{ }^{16} \mathrm{O}$ collision, the electronic energy transfer is identical to the one for the nuclear reaction, but the target potential is not, it is vice versa for the $\alpha+{ }^{13} \mathrm{C}$ collision.

In figure 9, the angular dependence of the capture probabilities for the same three collision systems is plotted. While the systems ${ }^{13} \mathrm{C}(\alpha, \alpha)$ and ${ }^{16} \mathrm{O}(\alpha, \alpha)$ show the strong decrease for $\theta \leqslant 90^{\circ}$ which is characteristic for elastic collisions, the capture probability for the ${ }^{16} \mathrm{O}(\mathbf{n}, \alpha)$ reaction depends only weakly on $\theta$. This may serve as a proof that the angular variation indeed is produced by the interference of the capture amplitudes from the incoming and outgoing parts of the collision.

\section{Conclusion}

We have formulated a theory for electron capture during reactive nuclear scattering, where the nuclear reaction is characterized by two channels, the initial and the final one, and where for each channel, the impulse approximation is used to calculate the atomic capture amplitudes. In correspondence to the case of elastic scattering, we were able to reduce the capture amplitude to a sum of terms, each of which factorizes into the nuclear reaction amplitude and an electronic transition amplitude. As a test of the theory, we have recalculated the capture probabilities for elastic $\mathrm{C}(\mathrm{p}, \mathrm{p})$ scattering and have found that the angular and energy dependence of the experimental data is fairly well reproduced. We attribute the discrepancies at the lower energies to the use of the transverse peaking approximation for the evaluation of the electronic amplitudes. Since one has a superposition of several contributions to the capture amplitude, some of them being calculated with and others without peaking approximations, the capture 
amplitude is particularly sensitive to the choice of approximations. An improvement could be achieved by doing an only one-dimensional peaking approximation in the potential terms and calculating the recoil exactly-at the expense of increasing com. puter time for this extra integral.

We have studied the dependence of the capture probability on the collision energy and the scattering angle for a variety of nuclear reactions induced by protons, deuterons and neutrons. The magnitude of interference structures in the energy dependence of $P(\theta)$ is strongly related to the ratio between the electronic energy transfer and the decay width of the compound nuclear state. The interference structures are very large when this ratio is above unity, as e.g. in the case of the ${ }^{32} S\left(p, p^{\prime}\right)$ reaction (where it is 2.2 ), and get weaker when the ratio decreases. For the reaction ${ }^{19} \mathrm{~F}(\mathrm{p}, \alpha)$ where the corresponding value is 0.25 , the excursion of $P(\theta)$ is at most a factor of 2 . For this reaction, we are able to describe the relative experimental dependence of the capture probability on the $\alpha$-particle energy rather well, but underestimate the absolute values by a factor of 3 . Part of this discrepancy can be explained if it is assumed that the electron cloud sticks to the centre-of-mass rather than to the recoiling ${ }^{16} \mathrm{O}$ nucleus, while the other part is attributed to the peaking approximation.

The absence of a slowly varying Coulomb contribution in the reaction amplitude allows for a much clearer display of the atomic interference effects than in the case of elastic resonances where additional nuclear interferences-which usually are not so obvious as in the ${ }^{32} \mathrm{~S}(\mathrm{p}, \mathrm{p})$ case-mix up with the atomic effects. Also, in contrast to the elastic scattering where the Coulomb amplitude is completely dominating at small scattering angles, interference structures in reactive scattering can be observed already at small angles. It should, however, be kept in mind that for angles well below $60^{\circ}$, the inelastic channel is only very weakly populated in the nuclear collision, and hence the number of channel- $b$ capture events is extremely low. The mere presence of a Breit-Wigner term in the reaction amplitude leads to a smooth decrease of $P(\theta)$ with $\theta$ for charged projectiles and a rather weak $\theta$-dependence of $P(\theta)$ for neutron impact. This confirms the interpretation that the angular variation of $P(\theta)$ results from an interference of the capture amplitudes from the incoming and the outgoing parts of the collision. Although these partial amplitudes are generally of the same order of magnitude for reactive scattering of charged projectiles, they are closer in modulus for elastic scattering. This leads to a larger variation of $P(\theta)$ with $\theta$ in the latter case.

Due to the strong dependence of the capture probability on the projectile charge and the collision velocity, $P(\theta)$ from reactive scattering is in the case of proton pick-up reactions (e.g. $(p, \alpha))$ strongly enhanced compared with the capture probability during elastic scattering. On the other hand, for large $Q$-values and hence ejectile velocities which by far exceed the impact velocity, the reactive collision leads to much smaller capture probabilities than the elastic collision. This is the case for the ${ }^{12} \mathrm{C}(\mathrm{d}, \mathrm{p})$ reaction with $Q \approx 2.7 \mathrm{MeV}$ - and vice versa for the ${ }^{32} \mathrm{~S}\left(\mathrm{p}, \mathrm{p}^{\prime}\right)$ reaction with $Q \simeq-2.2 \mathrm{MeV}$.

So far, the calculations have been restricted to capture from the target $\mathrm{K}$ shell. When the collision velocity falls below the electronic orbiting velocity of the target $K$ shell, capture from the $\mathrm{L}$ shell will come into play. The generalization of the capture theory to higher shells can be done in a similar way as in the case of elastic resonances (JAA). The basic effect of including $L$-shell capture in such systems will be a reduction of the interference structures, which is more severe, the lower the collision velocity and hence the smaller the energy transfer for an initial $L$ shell as compared with the energy transfer for $\mathrm{K}$-shell capture. The most promising candidate for an experimental investigation is the ${ }^{32} \mathbf{S}\left(\mathbf{p}, \mathbf{p}^{\prime}\right)$ reaction. For that system, L-shell capture is important, 
but the corresponding energy transfer of $\sim 1 \mathrm{keV}$ still compares well with the nuclear decay width such that the strong interference structures should remain visible.

\section{Acknowledgments}

I should like to thank P A Amundsen and E Horsdal Pedersen for helpful discussions, and $\mathrm{W}$ E Meyerhof for communicating his experimental results prior to publication. Financial support from the GSI Darmstadt is gratefully acknowledged.

\section{References}

Ajzenberg-Selove F 1986 Nucl. Phys. A 4601

-1987 Nucl. Phys. A 4751

Amundsen P A 1986 Unpublished notes

Amundsen P A and Aashamar K 1986 J. Phys. B: At. Mol. Phys. 191657

Amundsen P A and Jakubassa-Amundsen D H 1984a Phys. Rev. Lett. 53222

- 1984b J. Phys. B: At. Mol. Phys. 172671

Amundsen P A and Jakubassa-Amundsen D H 1991 Proc. 17th Int. Conf on Physics of Electronic and Atomic Collisions (Brisbane) Abstracts of contributed papers

Anholt R 1985 Atomic Inner-Shell Physics ed B Craseman (New York: Plenum) p 581

Baker O K, Meyerhof W E, Spooner D W and Stoller Ch 1988 Phys. Rev. Lett. 60913

Baldin A M, Goldanskij W I and Rosental I L 1963 Kinematik der Kernreaktionen (Berlin: Akademie) ch 8 Benn J, Dally E B, Müller H H, Prixley R E, Staub H H and Winkler H 1968 Nucl. Phys. A 106296

Blair J S and Anholt R 1982 Phys. Rev. A 25907

Dost M, Lorek R, Röhl S, Seidel J and Koenig W 1985 Phys. Rev. A 322077

Endt P M and van der Leun C 1973 Nucl. Phys. A 2141

Feagin J M and Kocbach L 1981 J. Phys. B: At. Mol. Phys. 144349

Heinz U 1987 Rep. Prog. Phys. 50145

Horsdal Pedersen E 1987 Nucl. Instrum. Methods B 24/25 130

Jakubassa-Amundsen D H 1984 Z. Phys. A 316161

1987 J. Phys. B: At. Mol. Phys. 20 L705 1990 Phys. Rev. Lett. 652247

Jakubassa-Amundsen D H and Amundsen P A 1985 J. Phys. B: At. Mol. Phys. 18757

Jeronymo J M F, Mani G S, Picard F and Sadeghi A 1963 Nucl. Phys. 43417

Joachain C J 1983 Quantum Collision Theory (Amsterdam: North-Holland) ch 14.2, 14.4, 18.2

Lane A M and Thomas R G 1958 Rev. Mod. Phys. 30257

McDowell M R C and Coleman J P 1970 Introduction to the Theory of Ion-Atom Collisions (Amsterdam: North-Holland) ch 8.6

McVoy K W and Weidenmüller H A 1982 Phys. Rev. A 251462

Olness J W, Haeberli W and Lewis H W 1958 Phys. Rev. 1121702

Scheurer J N, Baker O K and Meyerhof W E 1985 J. Phys. B: At. Mol. Phys. 18 L85

Taulbjerg K, Barrachina R O and Macek J H 1990 Phys. Rev. A 41207

Taylor J R 1972 Scattering Theory (New York: Wiley) ch 20d

Tomoda T 1984 Phys. Rev. A 29536

Tryti S, Holtebekk T and Rekstad J 1973 Nucl. Phys. A 201135

Végh L 1983 J. Phys. B: At. Mol. Phys. 164175 